\title{
NECESSARY AND SUFFICIENT CONDITIONS FOR THE TOPOLOGICAL CONJUGACY OF 3-DIFFEOMORPHISMS WITH HETEROCLINIC TANGENCIES
}

\author{
T. M. MITRYAKOVA AND O. V. POCHINKA
}

\begin{abstract}
In this paper we consider a class of three-dimensional diffeomorphisms that differ from gradient-like systems through the presence of heteroclinic tangencies. It is well known that such cascades are not structurally stable. However, here we find a complete system of topological invariants for a certain meaningful class of such diffeomorphisms.
\end{abstract}

\section{$\S 1$. InTRODUCTION AND STATEMENT OF THE RESUlTS}

Gradient-like flows are a classical object in regular dynamics. These are just the structurally stable flows generated by a vector field of the gradient of some Morse function (see, for example, 21]). On surfaces, such simple dynamics implies that the topological equivalence class 11 of a gradient-like flow is completely determined by the mutual position of the saddle separatrices. This fact was established in the classical papers by Andronov and Pontryagin [1] and Leontovich and Maĭer [10] for flows in a bounded part of the plane and was generalized to arbitrary surfaces by Peixoto [20], who expressed the information about the behaviour of separatrices using a distinguishing graph and proved that for a gradient-like flow the isomorphism class of its distinguishing graph is a complete topological invariant.

If the gradient flow generated on a surface by a Morse function is not structurally stable, then it has a pair of saddle equilibrium positions where the stable separatrix of one coincides with the unstable separatrix of the other. Such a violation of the condition of transversality of the intersection of the invariant manifolds of fixed points implies that in any $C^{1}$-neighbourhood of such a flow there exists a continuum of pairwise nonconjugate flows; this was first discovered by Palis [18]. A complete topological invariant of a nonrough gradient flow on a surface is in principle no longer combinatorial (like the distinguishing graph in the rough case) but necessarily has an analytic constituent describing a neighbourhood of the flow.

2010 Mathematics Subject Classification. Primary 37D20.

Key words and phrases. Heteroclinic tangencies, moduli of topological conjugacy, discrete dynamical systems.

This research was supported by the Programme for Basic Research of the National Research University Higher School of Economics in 2016 (project no. 98 "Topological methods in dynamics"), the Russian Science Foundation (project no. 15-01-03689-a), and the research work in accordance with the commission 2014/134 for performing state work in the sphere of scientific activity in the framework of the basic part of the state commission of the Ministry for Education and Science of Russia for 2014-2016 (Nizhnii Novgorod State University).

${ }^{1}$ Flows $f^{t}$ and $g^{t}$ defined on an $n$-manifold $M^{n}$ are said to be topologically equivalent if there exists a homeomorphism $h: M^{n} \rightarrow M^{n}$ taking the trajectories of the flow $f^{t}$ to trajectories of the flow $g^{t}$ preserving the orientation of motion along the trajectories. 
Morse-Smale diffeomorphisms (structurally stable diffeomorphisms with a finite nonwandering set) without heteroclinic points 2 give a discrete analogue of gradient-like flows. If a Morse-Smale diffeomorphism is defined on a surface and the invariant manifolds of its different saddle points are disjoint, then, as in the continuous case, its topological conjugacy class is determined by a graph similar to the Peixoto graph. This was proved by Bezdenezhnykh and Grines in [2] and [3]. Palis [18] also observed that the tangency of invariant manifolds of saddle points of a cascade along at least one orbit implies that the system is nonrough and, moreover, that a continuum of topologically nonconjugate diffeomorphisms exists in any $C^{1}$-neighbourhood of the system (modulus of topological conjugacy). If, in some neighbourhood of the diffeomorphism, it is possible to describe the set of equivalence classes by using finitely many parameters, then the diffeomorphism is said to have finitely many moduli of topological conjugacy. The term "modulus of topological conjugacy" was used in the papers of Shil'nikov and Gonchenko and corresponds to the term "modulus of stability", which is used in the Western literature.

Palis's paper led to a number of papers (see, for example, [11, 12, 13]) in which the structure of a neighbourhood of such a diffeomorphism was studied. In particular, in [13. necessary and sufficient conditions were found for a diffeomorphism of an orientable surface to have finitely many moduli of topological conjugacy describing all the topological conjugacy classes that belong to some neighbourhood of this diffeomorphism, and in [12] diffeomorphisms of $n$-dimensional manifolds with one orbit of one-sided heteroclinic tangency were considered and a classification of diffeomorphisms in a neighbourhood was given. Furthermore, a necessary condition for topological conjugacy of diffeomorphisms of $n$-dimensional manifolds containing one orbit of one-sided heteroclinic tangency was proved in [17. In 9] a necessary condition was proved for the topological conjugacy of diffeomorphisms defined on manifolds of dimension 3 which have finitely many orbits of heteroclinic tangency of two-dimensional invariant manifolds.

Mitryakova and Pochinka [15, 16] obtained a complete topological classification of diffeomorphisms on a surface that have a finite hyperbolic nonwandering set and are such that the saddle invariant manifolds of any two of its saddle points intersect (possibly, are tangent) over finitely many orbits. In this case, a complete topological invariant is a scheme consisting of finitely many tori with a set of smooth closed curves, where a real number is assigned to the point of tangency of every pair (the modulus of topological conjugacy).

In this paper necessary and sufficient conditions for topological conjugacy are obtained for diffeomorphisms of class $\Psi$ that are defined on smooth three-dimensional closed orientable manifolds $M^{3}$ and are such that any diffeomorphism $f \in \Psi$ has the following properties:

1) the nonwandering set $\Omega_{f}$ of the diffeomorphism $f$ consists of a finite number of hyperbolic points;

2) for different saddle points $p, q \in \Omega_{f}$ the intersection $W_{p}^{s} \cap W_{q}^{u}$ is not empty only in the case where $\operatorname{dim} W_{p}^{s}=\operatorname{dim} W_{q}^{u}=2$; in addition, it is transversal everywhere, except for, possibly, one orbit of nondegenerate one-sided tangency 3

\footnotetext{
${ }^{2} \mathrm{~A}$ diffeomorphism of a manifold is called a Morse-Smale diffeomorphism if its nonwandering set consists of finitely many hyperbolic periodic points whose invariant manifolds intersect transversally. If for different saddle periodic points $p, q$ of a Morse-Smale diffeomorphism the intersection $W_{p}^{s} \cap W_{q}^{u}$ is nonempty, then it is an infinite set. Furthermore, if $\operatorname{dim} W_{p}^{s}+\operatorname{dim} W_{q}^{u}=n$, then every point belonging to $W_{p}^{s} \cap W_{q}^{u}$ is called a heteroclinic point, and if $\operatorname{dim} W_{p}^{s}+\operatorname{dim} W_{q}^{u}>n$, then every connected component of $W_{p}^{s} \cap W_{q}^{u}$ is called a heteroclinic component. A Morse-Smale diffeomorphism is said to be gradient-like if its nonwandering set does not contain heteroclinic points.

${ }^{3}$ Let $N_{1}, N_{2}$ be two-dimensional submanifolds of a manifold $M^{3}$. A point $x \in N_{1} \cap N_{2}$ is called a point of nondegenerate one-sided tangency if there exists a chart $\left(U_{x}, \varphi_{x}\right)$ of the manifold $M^{3}$, where
} 
3 ) the saddle points of the diffeomorphism $f$ have $C^{2}$-linearizing neighbourhoods (see Definition 2.1 below).

The phase portrait of a diffeomorphism in the class $\Psi$ is shown in Figure 1. Note that when there are no heteroclinic points of tangency a diffeomorphism of class $\Psi$ is gradient-like. A complete topological classification of gradient-like diffeomorphisms on 3 -manifolds was obtained in [5] (see also Part 5 of the book [8]).

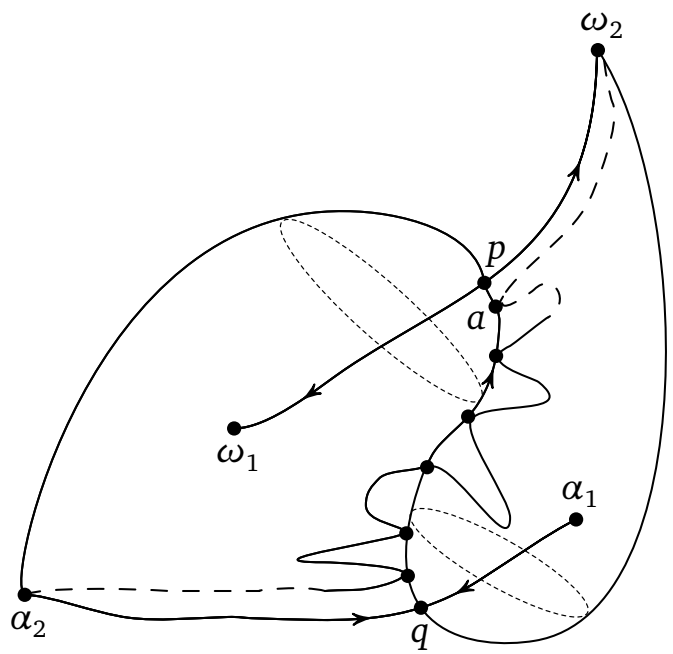

Figure 1. The phase portrait of a diffeomorphism in the class $\Psi$

In order to state our result, we introduce the following notation for a diffeomorphism $f \in \Psi$.

For $i \in\{0,1,2,3\}$, let $\Omega_{i}$ denote the subset of $\Omega_{f}$ consisting of the points $p$ such that $\operatorname{dim} W_{p}^{u}=i$. We set $A_{f}=W_{\Omega_{0} \cup \Omega_{1}}^{u}, R_{f}=W_{\Omega_{2} \cup \Omega_{3}}^{s}, V_{f}=M^{3} \backslash\left(A_{f} \cup R_{f}\right)$, and $\widehat{V}_{f}=V_{f} / f$. It follows from [7] that the sets $A_{f}, R_{f}, V_{f}$, and $\widehat{V}_{f}$ are connected, $\widehat{V}_{f}$ is a smooth closed 3-manifold, and the natural projection $p_{f}: V_{f} \rightarrow \widehat{V}_{f}$ is a covering which induces an epimorphism $\eta_{f}: \pi_{1}\left(\widehat{V}_{f}\right) \rightarrow \mathbb{Z}$ acting as follows. Let $\widehat{c}$ be a loop in $\widehat{V}_{f}$ such that $\widehat{c}(0)=\widehat{c}(1)=\widehat{x}$. By the monodromy theorem there exists a loop $c$ in $V_{f}$ starting at the point $x$ (where $c(0)=x$ ) which is a lifting of the path $\widehat{c}$, and there exists an element $k \in \mathbb{Z}$ such that $c(1)=f^{k}(x)$. Then the map $\eta_{f}: \pi_{1}\left(\widehat{V}_{f}\right) \rightarrow \mathbb{Z}$ takes $[\widehat{c}]$ to $k$.

We set

$$
\widehat{\mathbb{W}}_{f}^{s}=\bigcup_{p \in \Omega_{1}} \widehat{W}_{p}^{s} \quad \text { and } \quad \widehat{\mathbb{W}}_{f}^{u}=\bigcup_{p \in \Omega_{2}} \widehat{W}_{p}^{u}
$$

Every connected component $\widehat{W}_{p}^{\delta}, \delta \in\{s, u\}$, of the set $\widehat{\mathbb{W}}_{f}^{\delta}$ is an $\eta_{f}$-essential twodimensional torus or an $\eta_{f}$-essential Klein bottle on the manifold $\widehat{V}_{f}$ in the following sense. Let $j: \widehat{W}_{p}^{\delta} \rightarrow \widehat{V}_{f}$ be an inclusion, and $j_{*}: \pi_{1}\left(\widehat{W}_{p}^{\delta}\right) \rightarrow \pi_{1}\left(\widehat{V}_{f}\right)$ the induced homomorphism; then

$$
\eta_{f}\left(j_{*}\left(\pi_{1}\left(\widehat{W}_{p}^{\delta}\right)\right)\right) \neq\{0\} .
$$

Property 2 of the class $\Psi$ implies that connected components $\widehat{W}_{p}^{s} \subset \widehat{\mathbb{W}}_{f}^{s}$ and $\widehat{W}_{p}^{u} \subset \widehat{\mathbb{W}}_{f}^{u}$ either are disjoint, or intersect transversally, or intersect nontransversally, where the

$U_{x} \subset M^{3}$ is an open neighbourhood of the point $x$ and $\varphi_{x}: U_{x} \rightarrow \mathbb{R}^{3}$ is a $C^{2}$-diffeomorphism such that $\varphi_{x}(x)=(0,0,0), \varphi_{x}\left(N_{1} \cap U_{x}\right)=\left\{(x, y, z) \in \mathbb{R}^{3}: z=0\right\}, \varphi_{x}\left(N_{2} \cap U_{x}\right)=\left\{(x, y, z) \in \mathbb{R}^{3}: z=x^{2}+y^{2}\right\}$. 
condition of transversality at the intersection is violated at exactly one point, which is a point of nondegenerate one-sided tangency.

Let $\mathscr{A}$ denote the set of heteroclinic tangency points. For any point $a \in \mathscr{A}$ let $\sigma_{a}^{s}$ and $\sigma_{a}^{u}$ denote the saddle points such that $a$ belongs to the intersection of the invariant manifolds $W_{\sigma_{a}^{s}}^{s}$ and $W_{\sigma_{a}^{u}}^{u}$. Let $\mu_{a}\left(\lambda_{a}\right)$ denote the eigenvalue of the point $\sigma_{a}^{s}\left(\sigma_{a}^{u}\right)$ whose absolute value is greater than (less than) 1 . We set $\widehat{\mathscr{A}}=p_{f}(\mathscr{A})$. For $\widehat{a} \in \widehat{\mathscr{A}}$ we set $\Theta_{\widehat{a}}=\frac{\ln \left|\mu_{a}\right|}{\ln \left|\lambda_{a}\right|}$. Note that $\Theta_{\widehat{a}}$ is independent of the choice of the point in the set $p_{f}^{-1}(\widehat{a})$. We set $\widehat{C}_{f}=\left\{\Theta_{\widehat{a}}, \widehat{a} \in \widehat{\mathscr{A}}\right\}$.

Definition 1.1. The tuple $S_{f}=\left(\widehat{V}_{f}, \eta_{f}, \widehat{\mathbb{W}}_{f}^{s}, \widehat{\mathbb{W}}_{f}^{u}, \widehat{C}_{f}\right)$ is called the scheme of a diffeomorphism $f \in \Psi$.

Definition 1.2. The schemes $S_{f}$ and $S_{f^{\prime}}$ of diffeomorphisms $f, f^{\prime} \in \Psi$ are said to be equivalent if there exists a homeomorphism $\widehat{\varphi}: \widehat{V}_{f} \rightarrow \widehat{V}_{f^{\prime}}$ with the following properties:

1) $\eta_{f}=\eta_{f} \widehat{\varphi}_{*}$;

2) $\widehat{\varphi}\left(\widehat{\mathbb{W}}_{f}^{s}\right)=\widehat{\mathbb{W}}_{f}^{s}$, and $\widehat{\varphi}\left(\widehat{\mathbb{W}}_{f}^{u}\right)=\widehat{\mathbb{W}}_{f}^{u}$;

3) $\Theta_{\widehat{a}}=\Theta_{\widehat{\varphi}(\widehat{a})}$ for $\Theta_{\widehat{a}} \in \widehat{C}_{f}$.

Figure 2 depicts a three-dimensional annulus with touching cylinders embedded in it. Identifying the boundary spheres of the annulus results in a manifold $\mathbb{S}^{2} \times \mathbb{S}^{1}$. Gluing together the boundary circles of the cylinders produces touching tori. This shows the geometric constituent of the scheme of the diffeomorphism $f \in \Psi$, whose phase portrait is depicted in Figure 1, In this case the manifold $\widehat{V}_{f}$ is homeomorphic to $\mathbb{S}^{2} \times \mathbb{S}^{1}, \widehat{\mathbb{W}}_{f}^{s}=\widehat{W}_{p}^{s}$ and $\widehat{\mathbb{W}}_{f}^{u}=\widehat{W}_{q}^{u}$.

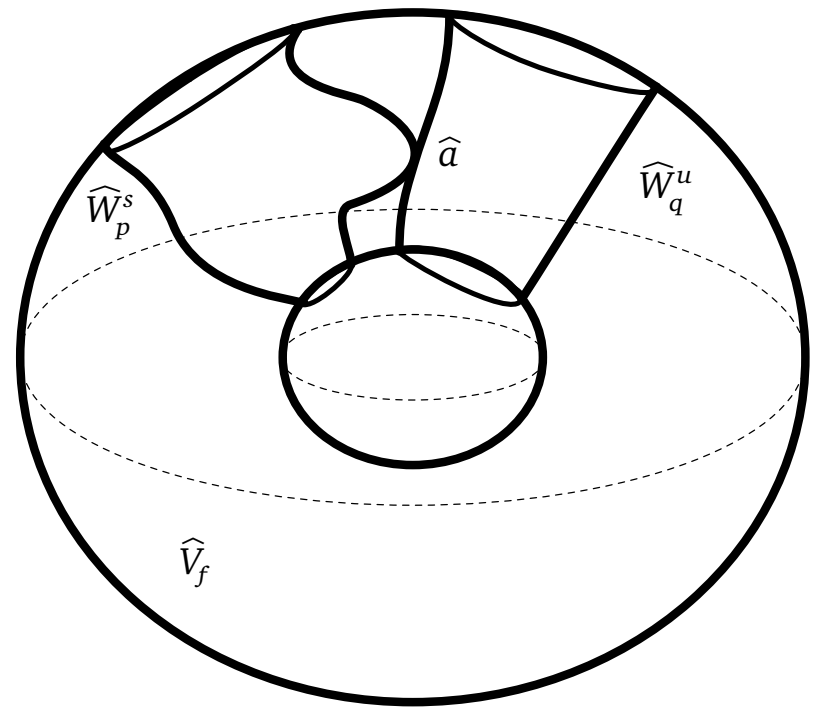

Figure 2. The geometric constituent of the scheme of the diffeomorphism $f \in \Psi$ whose phase portrait is depicted in Figure 1

The main result of this paper is the following theorem.

Theorem. Diffeomorphisms $f, f^{\prime} \in \Psi$ are topologically conjugate if and only if the schemes $S_{f}$ and $S_{f^{\prime}}$ are equivalent. 


\section{$\S 2$. LINEARIZING NEIGHBOURHOOD}

Suppose that a diffeomorphism $f$ belongs to the class $\Psi$ and $\sigma$ is one of its saddle points, which has period $m_{\sigma}$ and a two-dimensional stable manifold. Let $J_{\sigma}: \mathbb{R}^{3} \rightarrow \mathbb{R}^{3}$ denote the linear diffeomorphism defined by the Jordan form of the linear part of the diffeomorphism $f^{m_{\sigma}}$ in a neighbourhood of the point $\sigma$. The point $O(0,0,0)$ is a saddle point of the diffeomorphism $J_{\sigma}$ and has a $J_{\sigma}$-invariant neighbourhood $\mathscr{U}_{J_{\sigma}}$. Furthermore, $J_{\sigma}: \mathbb{R}^{3} \rightarrow \mathbb{R}^{3}$ and the $J_{\sigma}$-invariant neighbourhood $\mathscr{U}_{J_{\sigma}}$ of the saddle point $O(0,0,0)$ of $J_{\sigma}$ have one of the following three forms.

1) $J_{\sigma}\left(x_{1}, x_{2}, x_{3}\right)=\left(\lambda_{1} x_{1}, \lambda_{2} x_{2}, \mu x_{3}\right)$, where $0<\left|\lambda_{1}\right|,\left|\lambda_{2}\right|<1$ and $|\mu|>1$;

$$
\mathscr{U}_{J_{\sigma}}=\left\{\left(x_{1}, x_{2}, x_{3}\right) \in \mathbb{R}^{3}:\left(\left|x_{1}\right| \cdot\left|x_{3}\right|^{-\log _{|\mu|}\left|\lambda_{1}\right|}\right)^{2}+\left(\left|x_{2}\right| \cdot\left|x_{3}\right|^{-\log _{|\mu|}\left|\lambda_{2}\right|}\right)^{2}<1\right\}
$$

(see Figure 3).

2) $J_{\sigma}\left(x_{1}, x_{2}, x_{3}\right)=\left(\lambda x_{1}+x_{2}, \lambda x_{2}, \mu x_{3}\right)$, where $0<|\lambda|<1$ and $\mu>1$;

$$
\begin{aligned}
\mathscr{U}_{J_{\sigma}}=\left\{\left(x_{1}, x_{2}, x_{3}\right)\right. & \in \mathbb{R}^{3}:\left(x_{2}\left|x_{3}\right|^{-\log _{\mu}|\lambda|}\right)^{2} \\
& \left.+\left(x_{1}\left|x_{3}\right|^{-\log _{\mu}|\lambda|}-\frac{x_{2} \ln \left|x_{3}\right|}{|\lambda| \ln \mu} \cdot\left|x_{3}\right|^{-\log _{\mu}|\lambda|}\right)^{2}<1\right\} .
\end{aligned}
$$

3) $J_{\sigma}\left(x_{1}, x_{2}, x_{3}\right)=\left(\rho\left(x_{1} \cos \varphi-x_{2} \sin \varphi\right), \rho\left(x_{1} \sin \varphi+x_{2} \cos \varphi\right), \mu x_{3}\right)$ where $0<\rho<1$, $-\pi<\varphi \geq \pi$ and $\mu>1$;

$$
\mathscr{U}_{J_{\sigma}}=\left\{\left(x_{1}, x_{2}, x_{3}\right) \in \mathbb{R}^{3}: \sqrt{x_{1}^{2}+x_{2}^{2}}\left|x_{3}\right|^{-\log _{\mu} \rho}<1\right\} .
$$

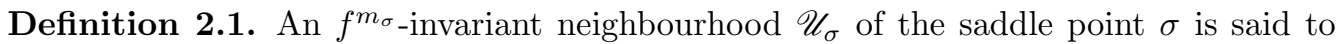
be $C^{2}$-linearizing if there exists a $C^{2}$-diffeomorphism $\psi_{\sigma}: \mathscr{U}_{\sigma} \rightarrow \mathscr{U}_{J_{\sigma}}$ conjugating the diffeomorphism $\left.f^{m_{\sigma}}\right|_{\mathscr{U}_{\sigma}}$ with the diffeomorphism $\left.J_{\sigma}\right|_{\mathscr{U}_{J_{\sigma}}}$.

A $C^{2}$-linearizing neighbourhood of a hyperbolic saddle point is constructed by applying the technique given in [9, Lemma 2] if the diffeomorphism is at least $C^{7}$-smooth and there are no resonances up to and including sixth orden 4 (see $[4$, Ch. $6, \S 5]$ or [22, Theorem 3.20]).

In the neighbourhood $\mathscr{U}_{J_{\sigma}}$ we define a pair of transversal foliations $\left(\mathscr{F}^{2}, \mathscr{F}^{1}\right)$ as follows:

- the leaves of the foliation $\mathscr{F}^{2}$ are the level sets of the function $\left(x_{1}, x_{2}, x_{3}\right) \mapsto x_{3}$ in $\mathscr{U}_{J_{\sigma}} ;$

- the leaves of the foliation $\mathscr{F}^{1}$ are the level sets of the function $\left(x_{1}, x_{2}, x_{3}\right) \mapsto\left(x_{1}, x_{2}\right)$ in $\mathscr{U}_{J_{\sigma}}$.

By means of the $C^{2}$-diffeomorphism $\psi_{\sigma}^{-1}$ the foliations $\mathscr{F}^{2}, \mathscr{F}^{1}$ induce $f^{m_{\sigma} \text {-invariant }}$ foliations $\mathscr{F}_{\sigma}^{2}, \mathscr{F}_{\sigma}^{1}$ on the linearizing neighbourhood $\mathscr{U}_{\sigma}$, which are called linearizing foliations. If $m_{\sigma}>1$, then by constructing a $C^{2}$-linearizing neighbourhood $\mathscr{U}_{\sigma}$ with a diffeomorphism $\psi_{\sigma}: \mathscr{U}_{\sigma} \rightarrow \mathscr{U}_{J_{\sigma}}$, for any $k=0, \ldots, m_{\sigma}-1$ we obtain a linearizing neighbourhood $U_{f^{k}(\sigma)}=f^{k}\left(U_{\sigma}\right)$ with the diffeomorphism $\psi_{f^{k}(\sigma)}=\psi_{\sigma} f^{-k}: \mathscr{U}_{f^{k}(\sigma)} \rightarrow$ $\mathscr{U}_{f^{k}(\sigma)}$ and linearizing foliations $\mathscr{F}_{f^{k}(\sigma)}^{2}, \mathscr{F}_{f^{k}(\sigma)}^{1}$. Throughout what follows we assume that linearizing neighbourhoods of saddle points of the same orbit are chosen in this compatible way.

\footnotetext{
${ }^{4}$ Let $p$ be a fixed hyperbolic point of a diffeomorphism $f: M^{n} \rightarrow M^{n}$, and let $\rho_{1}, \ldots, \rho_{n}$ be the eigenvalues of the Jacobi matrix $D_{p} f$. We say that a resonance of order $m \geq 2$ takes place at the point $p$ if there exist nonnegative numbers $m_{1}, \ldots, m_{n}$ such that $m=\sum_{k=1}^{n} m_{k}$ and the relation $\rho_{j}=\rho_{1}^{m_{1}} \rho_{2}^{m_{2}} \ldots \rho_{n}^{m_{n}}$ holds for some $j \in\{1, \ldots, n\}$.
} 


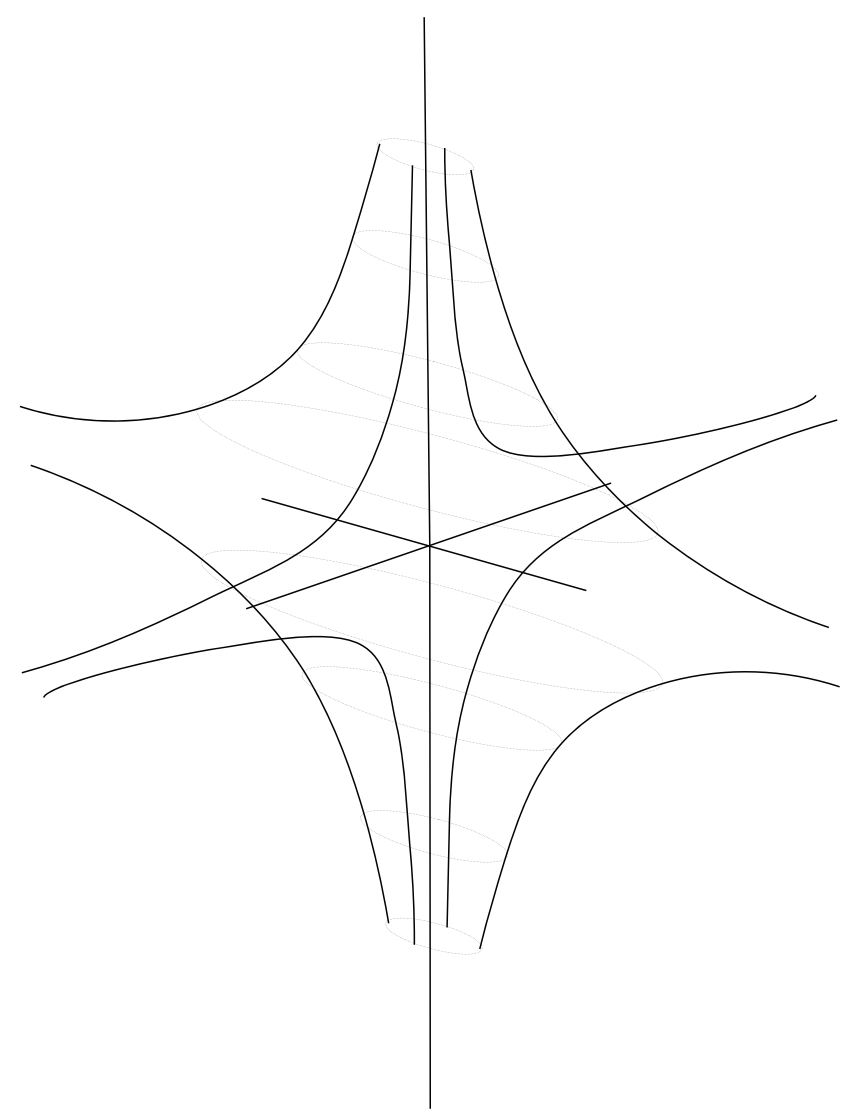

Figure 3. A linearizing neighbourhood $\mathscr{U}_{J_{\sigma}}$ for $J_{\sigma}\left(x_{1}, x_{2}, x_{3}\right)=\left(\lambda_{1} x_{1}, \lambda_{2} x_{2}, \mu x_{3}\right)$

By passing to the diffeomorphism $f^{-1}$ we define the $C^{2}$-linearizing neighbourhood with the linearizing foliations $\mathscr{F}_{\sigma}^{2}, \mathscr{F}_{\sigma}^{1}$ for a saddle point $\sigma$ with a two-dimensional unstable manifold. For any point $x \in \mathscr{U}_{\sigma}$ let $\mathscr{F}_{\sigma, x}^{2}, \mathscr{F}_{\sigma, x}^{1}$ denote the leaf of the foliation $\mathscr{F}_{\sigma}^{2}, \mathscr{F}_{\sigma}^{1}$ passing through the point $x$.

Let $a_{1}, \ldots, a_{k} \in \mathscr{A}$ be points that belong to pairwise different orbits such that their orbits comprise the whole set $\mathscr{A}$. By condition 2) in the description of the class $\Psi$, the point $a_{l}, l \in\{1, \ldots, k\}$, is a point of nondegenerate one-sided tangency of the manifolds $W_{\sigma_{a_{l}}^{s}, x}^{s}$ and $W_{\sigma_{a_{l}}^{u}, x}^{u}$, which are leaves of the $C^{2}$-smooth two-dimensional foliations $\mathscr{F}_{\sigma_{a_{l}}^{s}}^{2}$ and $\mathscr{F}_{\sigma_{a_{l}}^{u}}^{2}$. Then for any $l \in\{1, \ldots, k\}$ there exist a neighbourhood $\mathscr{U}_{a_{l}} \subset\left(\mathscr{U}_{\sigma_{a_{l}}^{s}} \cap \mathscr{U}_{\sigma_{a_{l}}}\right)$, a $C^{1}$-curve $\ell_{a_{l}} \subset \mathscr{U}_{a_{l}}$, and a two-dimensional foliation $B_{a_{l}}$ such that

1) the foliations $\mathscr{F}_{\sigma_{a_{l}}}^{2}$ and $\mathscr{F}_{\sigma_{a_{l}}^{u}}^{2}$ are transversal at every point of the set $\mathscr{U}_{a_{l}} \backslash \ell_{a_{l}}$, and the leaves $\mathscr{F}_{\sigma_{a_{l}}^{s}, x}^{2}$ and $\mathscr{F}_{\sigma_{l}^{u}}^{2}, x$ have nondegenerate one-sided tangency at every point $x \in \ell_{a_{l}}$;

2) the leaves of the foliations $F_{\sigma_{a_{l}}^{s}}^{2}$ and $F_{\sigma_{a_{l}}^{u}}^{2}$ are transversal to the foliation $B_{a_{l}}$ on $\mathscr{U}_{a_{l}} \backslash \ell_{a_{l}}$, and there exists a homeomorphism $\psi_{a_{l}}: U_{a_{l}} \rightarrow \mathbb{R}^{3}$ such that

$$
\psi_{a_{l}}\left(\ell_{a_{l}} \cap U_{a_{l}}\right)=O x_{3}, \quad B_{a_{l}}=\psi_{a_{l}}^{-1}(B),
$$

where

$$
B=\left\{\left(x_{1}, x_{2}, x_{3}\right) \in \mathbb{R}^{3} \backslash O x_{3}: x_{2}=k x_{1}, k \in \mathbb{R}\right\} \cup\left\{\left(x_{1}, x_{2}, x_{3}\right) \in \mathbb{R}^{3} \backslash O x_{3}: x_{1}=0\right\} ;
$$


3) $f^{k}\left(\mathscr{U}_{a_{l}}\right) \cap \mathscr{U}_{a_{l}}=\varnothing$ for any $k \in(\mathbb{Z} \backslash\{0\})$ (see Figure 41).

We set $L_{a_{l}}=\bigcup_{n \in \mathbb{Z}} f^{n}\left(\ell_{a_{l}}\right), L_{\mathscr{A}}=L_{a_{1}} \cup \cdots \cup L_{a_{k}}$, and $\mathscr{U}_{\mathscr{A}}=\mathscr{U}_{a_{1}} \cup \cdots \cup \mathscr{U}_{a_{k}}$.

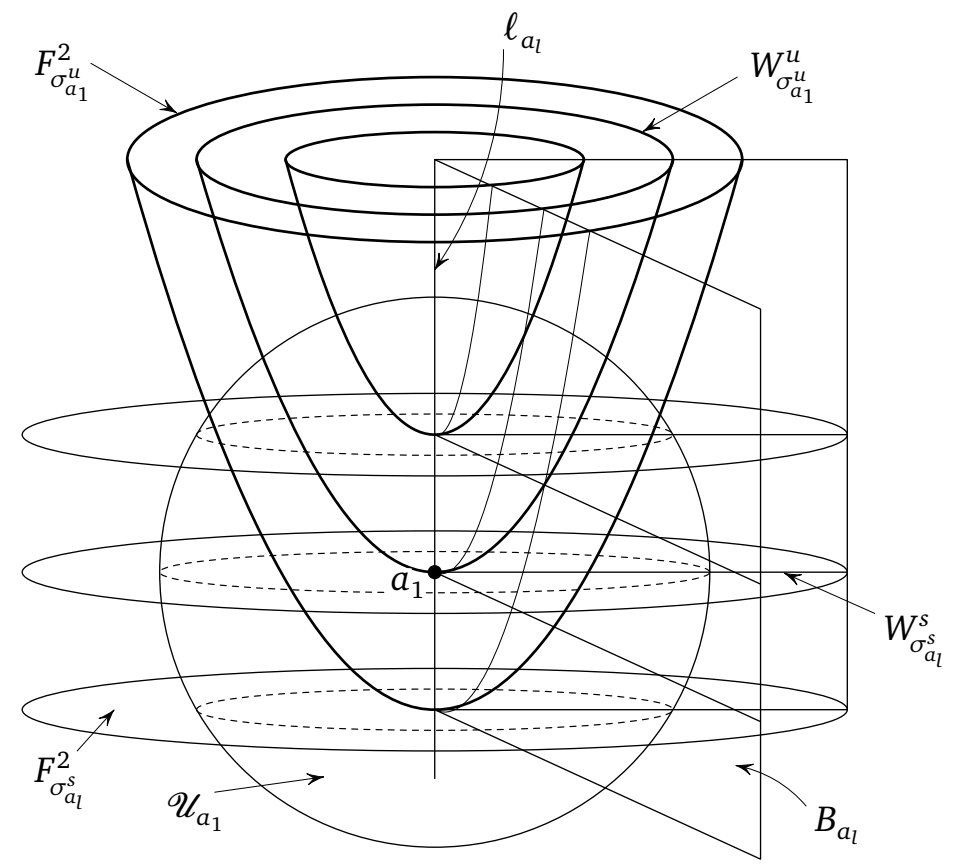

FIgURE 4. Foliations in a neighbourhood of a heteroclinic tangency point

\section{$\S$ 3. A COMpatible System of Neighbourhoods}

In this section we prove the existence of a compatible system of neighbourhoods of saddle points of any diffeomorphism in the class $\Psi$ in the sense of the following definition.

Definition 3.1. Let $f \in \Psi$. A system $\left\{U_{\sigma} \subset \mathscr{U}_{\sigma}, \sigma \in\left(\Omega_{1} \cup \Omega_{2}\right)\right\}$ of neighbourhoods of all saddle points is said to be compatible if their union is $f$-invariant and every neighbour-

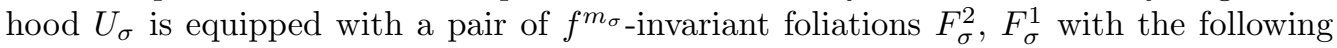
properties:

1) the foliation $F_{\sigma}^{2}$ is equal to $\mathscr{F}_{\sigma}^{2} \cap U_{\sigma}$;

2) the foliation $F_{\sigma}^{1}$ is a one-dimensional foliation that is transversal to the foliation $F_{\sigma}^{2}$ on $U_{\sigma} \backslash L_{\mathscr{A}}$, contains a one-dimensional manifold of the saddle point $\sigma$ as a leaf, and has singularities on the set $U_{\sigma} \cap L_{\mathscr{A}}$;

3) if $W_{\sigma_{1}}^{s} \cap W_{\sigma_{2}}^{u}=\varnothing$ for distinct saddle points $\sigma_{1}$ and $\sigma_{2}$, then $U_{\sigma_{1}} \cap U_{\sigma_{2}}=\varnothing$;

4) if $W_{\sigma_{1}}^{s} \cap W_{\sigma_{2}}^{u} \neq \varnothing$, then for any point $x \in\left(U_{\sigma_{1}} \cap U_{\sigma_{2}} \backslash L_{\mathscr{A}}\right)$ and a leaf $F_{\sigma_{1}, x}^{1}$ $\left(F_{\sigma_{2}, x}^{1}\right)$ of the foliation $F_{\sigma_{1}}^{1}\left(F_{\sigma_{2}}^{1}\right)$ passing through the point $x$, the condition $\left(F_{\sigma_{1}, x}^{1} \cap U_{\sigma_{2}}\right) \subset F_{\sigma_{2}, x}^{2}$ holds $\left(\left(F_{\sigma_{2}, x}^{1} \cap U_{\sigma_{1}}\right) \subset F_{\sigma_{1}, x}^{2}\right.$, respectively);

$5)$ any point $a_{l}, l \in\{1, \ldots, k\}$, has a neighbourhood $U_{a_{l}} \subset\left(U_{\sigma_{a_{l}}} \cap U_{\sigma_{a_{l}}^{u}}\right)$ in which every connected component of the intersection of a leaf of the foliation $F_{\sigma_{a_{l}}^{s}}^{2}\left(F_{\sigma_{a_{l}}^{u}}^{2}\right)$ with a leaf of the foliation $B_{a_{l}}$ is contained in a leaf of the foliation $F_{\sigma_{a_{l}}^{u}, a_{l}}^{1}\left(F_{\sigma_{a_{l}}^{s}}^{1}, a_{l}\right.$, respectively).

Theorem 3.1. For any diffeomorphism $f \in \Psi$ there exists a compatible system of neighbourhoods. 
Proof. We split the construction of a compatible system of neighbourhoods into steps.

Step 1. For any point $\sigma_{1} \in \Omega_{1}$ we set $\widehat{\mathscr{U}}_{\sigma_{1}}=p_{f}\left(\mathscr{U}_{\sigma_{1}}\right)$ and $\widehat{\mathscr{F}}_{\sigma_{1}}^{2}=p_{f}\left(\mathscr{F}_{\sigma_{1}}^{2}\right)$. For any point $\sigma_{2} \in \Omega_{2}$ we set $\widehat{\mathscr{U}_{\sigma_{2}}}=p_{f}\left(\mathscr{U}_{\sigma_{2}}\right)$ and $\widehat{\mathscr{F}}_{\sigma_{2}}^{2}=p_{f}\left(\mathscr{F}_{\sigma_{2}}^{2}\right)$. We set $\widehat{\mathscr{A}}=p_{f}(\mathscr{A})$. Every connected component of the sets $\widehat{\mathbb{W}}_{f}^{u}$ and $\widehat{\mathbb{W}}_{f}^{s}$ is a smooth compact surface. Since the intersection of the sets $\widehat{\mathbb{W}}_{f}^{u}$ and $\widehat{\mathbb{W}}_{f}^{s}$ is transversal everywhere apart from points in the set $\widehat{\mathscr{A}}$, the set $\widehat{H}=\widehat{\mathbb{W}}_{f}^{u} \cap \widehat{\mathbb{W}}_{f}^{s} \backslash \widehat{\mathscr{A}}$ consists of a finite number of simple closed curves $\widehat{\gamma}_{1}, \ldots, \widehat{\gamma}_{r}$, which are the projections of all the heteroclinic curves of the diffeomorphism $f$.

Any curve $\widehat{\gamma}_{l}, l=1, \ldots, r$, is contained in the intersection $\widehat{W}_{\sigma_{1}(l)}^{s} \cap \widehat{W}_{\sigma_{2}(l)}^{u}$ for some saddle points $\sigma_{1}(l) \in \Omega_{1}$ and $\sigma_{2}(l) \in \Omega_{2}$ (note that $\sigma_{1}\left(l_{1}\right)$ may coincide with $\sigma_{1}\left(l_{2}\right)$, just as the saddle $\sigma_{2}\left(l_{1}\right)$ may coincide with the saddle $\sigma_{2}\left(l_{2}\right)$ for distinct $\left.l_{1}, l_{2}\right)$. Since the curve $\widehat{\gamma}_{l}$ is compact, there exists a tubular neighbourhood $\mathscr{U}_{\widehat{\gamma}_{l}} \subset\left(\widehat{\mathscr{U}}_{\sigma_{1}(l)} \cap \widehat{\mathscr{U}}_{\sigma_{2}(l)}\right)$ with a foliation $\widehat{G}_{\widehat{\gamma}_{l}}=\left\{\widehat{d}_{x}^{l}, x \in \widehat{\gamma}_{l}\right\}$ consisting of discs transversal to the leaves of the foliations $\widehat{\mathscr{F}}_{\sigma_{1}}^{2}(l)$ and $\widehat{\mathscr{F}}_{\sigma_{2}(l)}^{2}$ such that the connected components of the intersection of the leaves of the foliations $\widehat{\mathscr{F}}_{\sigma_{1}(l)}^{2}, \widehat{\mathscr{F}}_{\sigma_{2}(l)}^{2}$ with the leaves of the foliation $\widehat{G}_{\widehat{\gamma}_{l}}$ form $C^{1}$-foliations $\widehat{F}_{\sigma_{2}(l), \widehat{\gamma}_{l}}^{1}$, $\widehat{F}_{\sigma_{1}(l), \widehat{\gamma}_{l}}^{1}$ on $\mathscr{U}_{\widehat{\gamma}_{l}}$ consisting of one-dimensional open arcs. Note that this fact will guarantee that properties 2) and 4) in Definition 3.1 hold in a neighbourhood of heteroclinic curves in what follows.

Let $U_{\widehat{\gamma}_{l}} \subset \mathscr{U}_{\widehat{\gamma}_{l}}$ be a neighbourhood of the curve $\widehat{\gamma}_{l}$ for which the projection

$$
\pi_{\sigma_{2}(l), \widehat{\gamma}_{l}}: U_{\widehat{\gamma}_{l}} \rightarrow \widehat{W}_{\sigma_{2}(l)}^{u} \quad\left(\pi_{\sigma_{1}(l), \widehat{\gamma}_{l}}: U_{\widehat{\gamma}_{l}} \rightarrow \widehat{W}_{\sigma_{1}(l)}^{s}\right),
$$

along the leaves of the foliation $\widehat{F}_{\sigma_{2}(l), \widehat{\gamma}_{l}}^{1}\left(\widehat{F}_{\sigma_{1}(l), \widehat{\gamma}_{l}}^{1}\right)$, is well defined. We set $U_{\widehat{H}}=U_{\widehat{\gamma}_{1}} \cup$ $\cdots \cup U_{\widehat{\gamma}_{r}}$. Let $\widehat{G}$ denote the two-dimensional $C^{1}$-foliation on $U(\widehat{H})$ composed of the discs of the foliations $\widehat{G}_{\widehat{\gamma}_{1}}, \ldots, \widehat{G}_{\widehat{\gamma}_{r}}$, let $\widehat{F}_{\Omega_{2}, \widehat{H}}^{1}\left(\widehat{F}_{\Omega_{1}, \widehat{H}}^{1}\right)$ denote the one-dimensional $C^{1}$-foliation composed of the leaves of the foliations $\widehat{F}_{\sigma_{2}(1), \widehat{\gamma}_{1}}^{1}, \ldots, \widehat{F}_{\sigma_{2}(r), \widehat{\gamma}_{r}}^{1}\left(\widehat{F}_{\sigma_{1}(1), \widehat{\gamma}_{1}}^{1}, \ldots, \widehat{F}_{\sigma_{1}(r), \widehat{\gamma}_{r}}^{1}\right)$, and let

$$
\pi_{\Omega_{2}, \widehat{H}}: U_{\widehat{H}} \rightarrow \widehat{\mathbb{W}}_{f}^{u} \quad\left(\pi_{\Omega_{1}, \widehat{H}}: U_{\widehat{H}} \rightarrow \widehat{\mathbb{W}}_{f}^{s}\right)
$$

denote the projection along the leaves of the foliation $\widehat{F}_{\Omega_{2}, \widehat{H}}^{1}\left(\widehat{F}_{\Omega_{1}, \widehat{H}}^{1}\right)$.

Step 2. For a point $\widehat{a}_{l} \in \widehat{\mathscr{A}}, l=1, \ldots, k$, we set $\mathscr{U}_{\widehat{a}_{l}}=p_{f}\left(\mathscr{U}_{a_{l}}\right), \ell_{\widehat{a}_{l}}=p_{f}\left(\ell_{a_{l}}\right)$, and $L_{\mathscr{A}}=p_{f}\left(L_{\mathscr{A}}\right)$. The point $\widehat{a}_{l}$ is a point of nondegenerate one-sided tangency of the surfaces $\widehat{W_{\sigma_{a_{l}}^{u}}^{u}}$ and $\widehat{W}_{\sigma_{a_{l}}^{s}}^{s}$, which are leaves of the two-dimensional $C^{2}$-foliations $\widehat{\mathscr{F}}_{\sigma_{a_{l}}^{s}}^{2}$ and $\widehat{\mathscr{F}}_{\sigma_{a_{l}}^{u}}^{2}$ that are transversal everywhere, except for points belonging to the $C^{1}$-curve $\ell_{\widehat{a}_{l}}$. We set $\widehat{B}_{a_{l}}=p_{f}\left(B_{a_{l}}\right)$. Then the connected components of the intersection of the leaves of the foliations $\widehat{\mathscr{F}_{\sigma_{l}}^{2}}, \widehat{\mathscr{F}_{\sigma_{l}}^{2}}$ with the leaves of the foliation $\widehat{B}_{\widehat{a}_{l}}=\psi_{\widehat{a}_{l}}^{-1}(B)$ form $C^{1}$-foliations $\widehat{F}_{\sigma_{a_{l}}^{u} \widehat{a}_{l}}^{1}$ and $\widehat{F}_{\sigma_{a_{l}}, \widehat{a}_{l}}^{1}$ on $\mathscr{U}_{\widehat{a}_{l}} \backslash \ell_{\widehat{a}_{l}}$ consisting of one-dimensional open arcs.

Let $K_{\widehat{a}_{l}}^{u} \subset \mathscr{U}_{\widehat{a}_{l}}\left(K_{\widehat{a}_{l}}^{s} \subset \mathscr{U}_{\widehat{a}_{l}}\right)$ be the union of the leaves of the foliation $\widehat{F}_{\sigma_{a_{l}}^{s}, \widehat{a}_{l}}^{1}\left(\widehat{F}_{\sigma_{a_{l}}^{u}}^{1}, \widehat{a}_{l}\right)$ that have nonempty intersection with $\widehat{W}_{\sigma_{a_{l}}^{s}}^{s}\left(\widehat{W}_{\sigma_{a_{l}}^{u}}^{u}\right.$, respectively) (see Figure 5 ). Then on the set $K_{\widehat{a}_{l}}^{u} \subset \mathscr{U}_{\widehat{a}_{l}}\left(K_{\widehat{a}_{l}}^{s} \subset \mathscr{U}_{\widehat{a}_{l}}\right)$, the projection

$$
\pi_{\sigma_{a_{l}}^{s}, \widehat{a}_{l}}: K_{\widehat{a}_{l}}^{u} \rightarrow \widehat{W}_{\sigma_{a_{l}}^{s}}^{s} \quad\left(\pi_{\sigma_{a_{l}}^{u}, \widehat{a}_{l}}: K_{\widehat{a}_{l}}^{s} \rightarrow \widehat{W}_{\sigma_{a_{l}}^{u}}^{u}\right)
$$

along the leaves of the foliation $\widehat{F}_{\sigma_{a_{l}}^{s}}^{1}, \widehat{a}_{l}\left(\widehat{F}_{\sigma_{a_{l}}^{u}}^{1}, \widehat{a}_{l}\right)$ is well defined. We choose a connected component of the intersection of a leaf of the foliation $\widehat{\mathscr{F}}_{a_{l}^{u}}^{2}\left(\widehat{\mathscr{F}}_{\sigma_{a_{l}}^{s}}^{2}\right)$ with the interior of 


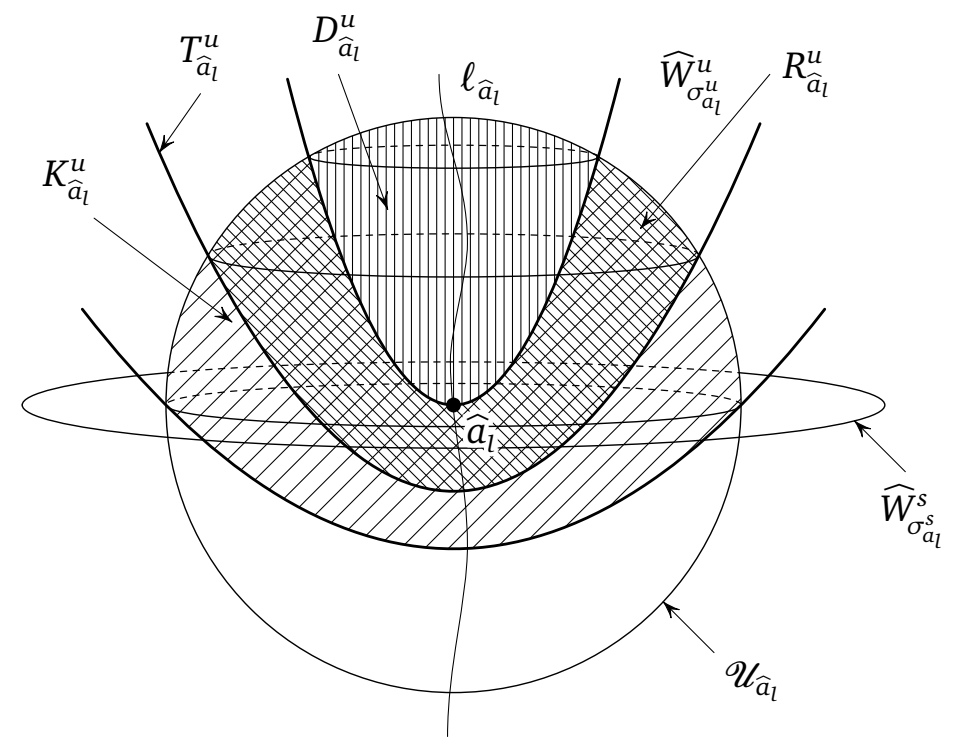

Figure 5. The neighbourhood $\mathscr{U}_{\widehat{a}_{l}}$

the set $K_{\widehat{a}_{l}}^{u}\left(K_{\widehat{a}_{l}}^{s}\right)$, and denote it by $T_{\widehat{a}_{l}}^{u}\left(T_{\widehat{a}_{l}}^{s}\right)$. Let $R_{\widehat{a}_{l}}^{u}\left(R_{\widehat{a}_{l}}^{s}\right)$ denote the closure of the connected component of the set $K_{\widehat{a}_{l}}^{u} \backslash c l T_{\widehat{a}_{l}}^{u}\left(K_{\widehat{a}_{l}}^{s} \backslash c l T_{\widehat{a}_{l}}^{s}\right)$ that has nonempty intersection with $\widehat{W}_{\sigma_{a_{l}}^{u}}^{u}\left(\widehat{W}_{\sigma_{a_{l}}^{s}}^{s}\right.$, respectively) (the set with the double hatching in Figure [5). Let $D_{\widehat{a}_{l}}^{u}$ $\left(D_{\widehat{a}_{l}}^{s}\right)$ denote the closure of the connected component of the set $\mathscr{U}_{\widehat{a}_{l}} \backslash \widehat{W}_{\sigma_{a_{l}}^{u}}^{u}\left(\mathscr{U}_{\widehat{a}_{l}} \backslash \widehat{W}_{\sigma_{a_{l}}^{s}}^{s}\right)$ the interior of which is disjoint from $\widehat{W}_{\sigma_{a_{l}}^{s}}^{s}\left(\widehat{W}_{\sigma_{a_{l}}^{u}}^{u}\right.$, respectively) (the set with the vertical hatching in Figure 5).

We set $K_{\widehat{A}}^{u}=K_{\widehat{a}_{1}}^{u} \cup \cdots \cup K_{\widehat{a}_{k}}^{u}, R_{\widehat{A}}^{u}=R_{\widehat{a}_{1}}^{u} \cup \cdots \cup R_{\widehat{a}_{k}}^{u}, D_{\widehat{A}}^{u}=D_{\widehat{a}_{1}}^{u} \cup \cdots \cup D_{\widehat{a}_{k}}^{u}$, $K_{\widehat{\mathscr{A}}}^{s}=K_{\widehat{a}_{1}}^{s} \cup \cdots \cup K_{\widehat{a}_{k}}^{s}, R_{\widehat{\mathscr{A}}}^{s}=R_{\widehat{a}_{1}}^{s} \cup \cdots \cup R_{\widehat{a}_{k}}^{s}$, and $D_{\widehat{\mathscr{A}}}^{s}=D_{\widehat{a}_{1}}^{s} \cup \cdots \cup D_{\widehat{a}_{k}}^{s}$. Let $\widehat{B}$ denote the two-dimensional $C^{1}$-foliation on $\mathscr{U}_{\hat{A}} \backslash L_{\hat{\mathscr{A}}}$ composed of the leaves of the foliations $\widehat{B}_{\widehat{a}_{1}}, \ldots, \widehat{B}_{\widehat{a}_{k}}$, let $\widehat{F}_{\Omega_{2}, \widehat{A}}^{1}$ (respectively, $\widehat{F}_{\Omega_{1}, \widehat{A}}^{1}$ ) denote the one-dimensional $C^{1}$-foliation composed of the leaves of the foliations $\widehat{F}_{\sigma_{a_{1}}^{u}, \widehat{a}_{1}}^{1}, \ldots, \widehat{F}_{\sigma_{a_{r}}^{u}}^{1}, \widehat{a}_{r}\left(\widehat{F}_{\sigma_{a_{1}}^{s}}^{1}, \widehat{a}_{1}, \ldots, \widehat{F}_{\sigma_{a_{r}}^{s}}^{1}, \widehat{a}_{r}\right)$, and let $\pi_{\Omega_{2}, \hat{\mathscr{A}}}: K_{\widehat{\mathscr{A}}}^{u} \rightarrow \widehat{\mathbb{W}}_{f}^{u}\left(\pi_{\Omega_{1}, \widehat{\mathscr{A}}}: K_{\widehat{\mathscr{A}}}^{s} \rightarrow \widehat{\mathbb{W}}_{f}^{s}\right)$ denote the projection along the leaves of the foliation $\widehat{F}_{\Omega_{2}, \hat{A}}^{1}\left(\widehat{F}_{\Omega_{1}, \hat{A}}^{1}\right)$.

Step 3. We construct pairwise disjoint $f$-invariant neighbourhoods $U_{\sigma}, \sigma \in \Omega_{2}$, equipped with one-dimensional $f$-invariant $C^{1}$-foliations $F_{\sigma}^{1}$ that have properties 2) and 4) from Definition 3.1 in some neighbourhood of heteroclinic curves and heteroclinic tangency points.

The set $\widehat{W}_{\sigma}^{u}$ is a smooth submanifold of the manifold $\widehat{V}_{f}$ homeomorphic to a twodimensional torus or the Klein bottle. Then there exists a tubular neighbourhood $\widehat{N}_{\sigma}^{u}$ of the manifold $\widehat{W}_{\sigma}^{u}$ with the projection $\pi_{\sigma}: \widehat{N}_{\sigma}^{u} \rightarrow \widehat{W}_{\sigma}^{u}$ along the one-dimensional leaves of the foliation $\left\{\widehat{I}_{\sigma, \widehat{x}}, \widehat{x} \in \widehat{W}_{\sigma}^{u}\right\}$. We set $P_{\sigma}=\widehat{N}_{\sigma}^{u} \cap U_{\widehat{H}}, Q_{\sigma}=\widehat{N}_{\sigma}^{u} \cap K_{\widehat{\mathscr{A}}}^{u}$, and $Q_{\sigma}^{\prime}=\widehat{N}_{\sigma}^{u} \cap R_{\widehat{\mathscr{A}}}^{u}$. Let $\pi_{\sigma}^{\prime}: P_{\sigma} \cup Q_{\sigma} \rightarrow \widehat{W}_{\sigma}^{u}$ denote the map coinciding with $\pi_{\Omega_{2}, \widehat{H}}$ on $P_{\sigma}$ and coinciding with $\pi_{\Omega_{2}, \hat{A}}$ on $Q_{\sigma}$. Let $P_{\sigma}^{\prime} \subset P_{\sigma}$ be a neighbourhood of the set $\widehat{W}_{\sigma}^{u} \cap \widehat{H}$ such that the set $P_{\sigma} \backslash P_{\sigma}^{\prime}$ is fibred by the leaves of the foliation $\widehat{F}_{\Omega_{2}, \widehat{H}}^{1}$. Let $\phi: \widehat{N}_{\sigma}^{u} \backslash D_{\widehat{\mathscr{A}}}^{u} \rightarrow[0,1]$ be a 
smooth function with support on $P_{\sigma} \cup Q_{\sigma}$ that is equal to 1 on $P_{\sigma}^{\prime} \cup Q_{\sigma}^{\prime}$. Since the surface $\widehat{W}_{\sigma}^{u}$ has the structure of a Riemannian manifold $\mathbb{R}^{2} / G$, where $G$ is a group of isometries acting freely and discontinuously 5 it follows that the following formula gives a well-defined $C^{1}$-retraction $q_{\sigma}: \widehat{N}_{\sigma}^{u} \backslash D_{\widehat{\Omega}}^{u} \rightarrow \widehat{W}_{\sigma}^{u}$ :

$$
q_{\sigma}(x)=(1-\phi(x)) \pi_{\sigma}(x)+\phi(x) \pi_{\sigma}^{\prime}(x) .
$$

Since $q_{\sigma}(x)=x$ for $x \in \widehat{W}_{\sigma}^{u}$, there exists a neighbourhood $\widetilde{U}_{\sigma}^{u} \subset \widehat{N}_{\sigma}^{u}$ of the manifold $\widehat{W}_{\sigma}^{u}$ with the one-dimensional foliation $\widetilde{F}_{\sigma}^{1}$ composed of the $\operatorname{arcs}\left\{\left(q_{\sigma}\right)^{-1}(x) \cap \widetilde{U}_{\sigma}^{u}, x \in\right.$ $\left.\widehat{W}_{\sigma}^{u} \backslash Q_{\sigma}^{\prime}\right\}$, the $\operatorname{arcs} L_{\hat{A}} \cap \widetilde{U}_{\sigma}^{u}$, and on the remaining set composed of the leaves of the foliation $\widehat{F}_{\Omega_{2}, \hat{A}}^{1} \cap \widetilde{U}_{\sigma}^{u}$. We set $S_{\sigma}=\widetilde{U}_{\sigma}^{u} \cap\left(P_{\sigma}^{\prime} \cup Q_{\sigma}^{\prime} \cup D_{\widehat{A}}^{u}\right)$ and $S_{\Omega_{2}}=\bigcup_{\sigma \in \Omega_{2}} S_{\sigma}$.

We perform similar constructions with similar notation for points of the set $\Omega_{1}$. For points $\sigma \in \Omega_{2}\left(\sigma \in \Omega_{1}\right)$ we choose neighbourhoods $\widehat{U}_{\sigma}^{u} \subset \widetilde{U}_{\sigma}^{u}\left(\widehat{U}_{\sigma}^{s} \subset \widetilde{U}_{\sigma}^{s}\right)$ such that the union of these neighbourhoods $\widehat{U}_{\Omega_{2}}^{u}=\bigcup_{\sigma \in \Omega_{2}} \widehat{U}_{\sigma}^{u}\left(\widehat{U}_{\Omega_{1}}^{s}=\bigcup_{\sigma \in \Omega_{1}} \widehat{U}_{\sigma}^{s}\right.$, respectively) has the property that $\widehat{U}_{\Omega_{2}}^{u} \cap \widehat{U}_{\Omega_{1}}^{s} \subset\left(S_{\Omega_{2}} \cap S_{\Omega_{1}}\right)$.

For $\sigma \in \Omega_{2}\left(\sigma \in \Omega_{1}\right)$ we set $\widehat{F}_{\sigma}^{1}=\widetilde{F}_{\sigma}^{1} \cap \widehat{U}_{\sigma}^{u}\left(\widehat{F}_{\sigma}^{1}=\widetilde{F}_{\sigma}^{1} \cap \widehat{U}_{\sigma}^{s}\right)$, and $U_{\mathscr{O}_{\sigma}}=p_{f}^{-1}\left(\widehat{U}_{\sigma}^{u}\right) \cup W_{\mathscr{O}_{\sigma}}^{s}$ $\left(U_{\mathscr{O}_{\sigma}}=p_{f}^{-1}\left(\widehat{U}_{\sigma}^{s}\right) \cup W_{\mathscr{O}_{\sigma}}^{u}\right)$, where $\mathscr{O}_{\sigma}$ is the orbit of the point $\sigma$. Let $F_{\mathscr{O}_{\sigma}}^{1}$ denote the foliation on $U_{\mathscr{O}_{\sigma}}$ consisting of the connected components of the pre-images under $p_{f}$ of the leaves of the foliation $\widehat{F}_{\sigma}^{1}$ and the stable (unstable) manifolds $W_{\mathscr{O}_{\sigma}}^{s}\left(W_{\mathscr{O}_{\sigma}}^{u}\right.$, respectively). Let $U_{\sigma}$ be a connected component of the set $U_{\mathscr{O}_{\sigma}}$. Then the restriction $F_{\sigma}^{1}$ of the foliation $F_{\mathscr{O}_{\sigma}}^{1}$ to $U_{\sigma}$ is the one we are looking for.

\section{$\S 4$. NeCESSARY AND SUfFiCiENT CONDitions FOR TOPOLOGICAL CONJUGACY}

Theorem 4.1. Diffeomorphisms $f, f^{\prime} \in \Psi$ are topologically conjugate if and only if the schemes $S_{f}$ and $S_{f^{\prime}}$ are equivalent.

Proof. The necessity for the geometric constituents of the schemes to be homeomorphic is proved as in [6. Lemma 1.3.6], and that it is necessary that the analytic parameters of the schemes be equal is proved in [9, Theorem 1]. We now prove the sufficiency of the conditions of the theorem, that is, we construct a homeomorphism conjugating the diffeomorphisms $f$ and $f^{\prime}$ in the class $\Psi$ (the scheme of construction is close to the construction of a conjugating homeomorphism for diffeomorphisms of surfaces with the same modulus of stability found in [14]).

Suppose that the schemes $S_{f}$ and $S_{f^{\prime}}$ of diffeomorphisms $f, f^{\prime} \in \Psi$ are equivalent. Then by Definition 1.2 there exists a homeomorphism $\widehat{\varphi}: \widehat{V}_{f} \rightarrow \widehat{V}_{f^{\prime}}$ such that

1) $\eta_{f}=\eta_{f} \widehat{\varphi}_{*}$;

2) $\widehat{\varphi}\left(\widehat{\mathbb{W}}_{f}^{s}\right)=\widehat{\mathbb{W}}_{f^{\prime}}^{s}$ and $\widehat{\varphi}\left(\widehat{\mathbb{W}}_{f}^{u}\right)=\widehat{\mathbb{W}}_{f^{\prime}}^{u}$;

3) $\Theta_{\widehat{a}}=\Theta_{\widehat{\varphi}(\widehat{a})}$ for $\Theta_{\widehat{a}} \in \widehat{C}_{f}$.

In constructing a conjugating homeomorphism we make key use of the existence of a compatible system of neighbourhoods for a Morse-Smale diffeomorphism (see Definition 3.1). We divide the construction of a homeomorphism $h: M^{3} \rightarrow M^{3}$ conjugating the diffeomorphisms $f, f^{\prime}$ into steps.

In Step 1 we prove the existence of a lifting $\varphi: V_{f} \rightarrow V_{f^{\prime}}$ of a homeomorphism $\widehat{\varphi}$ conjugating the diffeomorphisms $\left.f\right|_{V_{f}}$ and $\left.f^{\prime}\right|_{V_{f^{\prime}}}$, which is uniquely extended to the set $\Omega_{1} \cup \Omega_{2}$.

\footnotetext{
${ }^{5}$ If $\widehat{W}_{\sigma}^{u}$ is a torus, then we find that $G$ is a group of diffeomorphisms of the plane $\mathbb{R}^{2}$ with generators $a\left(x_{1}, x_{2}\right)=\left(x_{1}+1, x_{2}\right)$ and $b\left(x_{1}, x_{2}\right)=\left(x_{1}, x_{2}+1\right)$. If $\widehat{W}_{\sigma}^{u}$ is a Klein bottle, then $G$ is a group of diffeomorphisms of the plane $\mathbb{R}^{2}$ with generators $c\left(x_{1}, x_{2}\right)=\left(x_{1}+1, x_{2}\right)$ and $d\left(x_{1}, x_{2}\right)=\left(1-x_{1}, x_{2}+1 / 2\right)$.
} 
In Step 2 we construct a homeomorphism $\varphi_{\Omega_{1}}^{u}: W_{\Omega_{1}}^{u} \rightarrow W_{\Omega_{1}^{\prime}}^{u}$ conjugating the diffeomorphisms $\left.f\right|_{W_{\Omega_{1}}^{u}}$ and $\left.f^{\prime}\right|_{W_{\Omega_{1}^{\prime}}^{u}}$. Here, the map $\varphi_{\Omega_{1}}^{u}$ is composed of the maps $\varphi_{\sigma}^{u}: W_{\sigma}^{u} \rightarrow$ $W_{\sigma^{\prime}}^{u}, \sigma \in \Omega_{1}$, which are uniquely defined by the eigenvalues $\mu_{\sigma}$ of the map $J_{\sigma}$ (eigenvalues $\mu_{\sigma^{\prime}}$ of $\left.J_{\sigma^{\prime}}\right)$ that are greater than 1 in absolute value.

In Step 3 we construct a homeomorphism $\varphi_{\Omega_{2}}^{s}: W_{\Omega_{2}}^{s} \rightarrow W_{\Omega_{2}^{\prime}}^{s}$ conjugating the diffeomorphisms $\left.f\right|_{W_{\Omega_{2}}^{s}}$ and $\left.f^{\prime}\right|_{W_{\Omega_{2}^{\prime}}^{s}}$. Here, the map $\varphi_{\Omega_{1}}^{s}$ is composed of the maps $\varphi_{\sigma}^{s}: W_{\sigma}^{s} \rightarrow$ $W_{\sigma^{\prime}}^{s}, \sigma \in \Omega_{2}$, which are uniquely defined by the eigenvalues $\lambda_{\sigma}\left(\lambda_{\sigma^{\prime}}\right)$ of the map $J_{\sigma}$ $\left(J_{\sigma^{\prime}}\right)$ that are less than 1 in absolute value, and by the map of passing from one set of linearizing coordinates to another in a neighbourhood of a heteroclinic tangency point.

In Step 4, using the map $\varphi_{\Omega_{1}}^{u}$, we modify the homeomorphism $\varphi$ to a homeomorphism $\varphi_{0}: V_{f} \rightarrow V_{f^{\prime}}$ conjugating the diffeomorphisms $\left.f\right|_{V_{f}}$ and $\left.f^{\prime}\right|_{V_{f^{\prime}}}$ that coincides with $\varphi$ outside a neighbourhood $V_{\mathscr{A}}^{2}$ of the set of heteroclinic tangency points and takes compatible leaves of the diffeomorphism $f$ to compatible leaves of the diffeomorphism $f^{\prime}$ in a neighbourhood $V_{\mathscr{A}}^{1} \subset V_{\mathscr{A}}^{2}$ of the set of heteroclinic tangency points.

In Step 5 we modify the homeomorphism $\varphi_{0}$ to a homeomorphism $\varphi_{1}: V_{f} \rightarrow V_{f^{\prime}}$ that conjugates the diffeomorphisms $\left.f\right|_{V_{f}}$ and $\left.f^{\prime}\right|_{V_{f^{\prime}}}$, takes compatible two-dimensional stable foliations of the diffeomorphism $f$ in the neighbourhood $W_{\Omega_{1}}^{u}$ to compatible twodimensional stable foliations of the diffeomorphism $f^{\prime}$ in the neighbourhood $W_{\Omega_{1}^{\prime}}^{u}$, and can be continuously extended to the set $W_{\Omega_{1}}^{u}$ by a homeomorphism $\varphi_{\Omega_{1}}^{u}$.

In Step 6 the homeomorphism $\widehat{\varphi}_{1}$ is modified to a homeomorphism $\varphi_{2}: V_{f} \rightarrow V_{f^{\prime}}$ that conjugates the diffeomorphisms $\left.f\right|_{V_{f}}$ and $\left.f^{\prime}\right|_{V_{f^{\prime}}}$, takes compatible two-dimensional foliations of the diffeomorphism $f$ in the neighbourhood $W_{\Omega_{2}}^{s} \cup W_{\Omega_{1}}^{u}$ to compatible twodimensional foliations of the diffeomorphism $f^{\prime}$ in the neighbourhood $W_{\Omega_{2}^{\prime}}^{s} \cup W_{\Omega_{1}^{\prime}}^{u}$, and can be continuously extended to the set $W_{\Omega_{1}}^{u}$ by the homeomorphism $\varphi_{\Omega_{1}}^{u}$, and to the set $W_{\Omega_{2}}^{s}$ by the homeomorphism $\varphi_{\Omega_{2}}^{s}$. The homeomorphism $\varphi_{2}$ thus constructed can be uniquely extended on the set of nodal points to a homeomorphism $h$, which is the one required.

Step 1. It follows from condition 1) in Definition 1.2 concerning the equivalence of schemes that there exists a homeomorphism $\varphi: V_{f} \rightarrow V_{f^{\prime}}$ conjugating the restriction of the diffeomorphism $f$ to $V_{f}$ with the restriction of the diffeomorphism $f^{\prime}$ to $V_{f^{\prime}}$ such that $\widehat{\varphi}=p_{f^{\prime}} \varphi p_{f}^{-1}$ (see, for example, [6, Proposition 1.2.4]). Thus, we have a conjugating homeomorphism on the set $M^{3} \backslash\left(W_{\Omega_{0} \cup \Omega_{1}}^{u} \cup W_{\Omega_{2} \cup \Omega_{3}}^{s}\right)$.

Due to condition 2) in Definition [1.2, for any point $\sigma \in \Omega_{1}\left(\sigma \in \Omega_{2}\right)$ there exists a point $\sigma^{\prime} \in \Omega_{1}^{\prime}\left(\sigma^{\prime} \in \Omega_{2}^{\prime}\right)$ such that $\varphi\left(W_{\sigma}^{s} \backslash \sigma\right)=W_{\sigma^{\prime}}^{s} \backslash \sigma^{\prime}\left(\varphi\left(W_{\sigma}^{u} \backslash \sigma\right)=W_{\sigma^{\prime}}^{u} \backslash \sigma^{\prime}\right)$. We extend the homeomorphism $\varphi$ to the set $\Omega_{1} \cup \Omega_{2}$ by setting $\varphi(\sigma)=\sigma^{\prime}$ for $\sigma \in\left(\Omega_{1} \cup \Omega_{2}\right)$.

Step 2. We define a conjugating homeomorphism $\varphi^{u}: W_{\Omega_{1}}^{u} \rightarrow W_{\Omega_{1}^{\prime}}^{u}$

Let $\sigma \in \Omega_{1}$ and $\sigma^{\prime}=\varphi(\sigma)$, and let $\psi_{\sigma}$ and $\psi_{\sigma^{\prime}}$ be linearizing diffeomorphisms on linearizing neighbourhoods $\mathscr{U}_{\sigma}$ and $\mathscr{U}_{\sigma^{\prime}}$ (see Definition 2.1). For any point $w \in W_{\sigma}^{u}$ $\left(w^{\prime} \in W_{\sigma^{\prime}}^{u}\right)$ the point $\psi_{\sigma}(w)\left(\psi_{\sigma^{\prime}}\left(w^{\prime}\right)\right)$ has coordinates $\left(0,0, w_{3}\right)\left(\left(0,0, w_{3}^{\prime}\right)\right)$. We set $r=\frac{\ln \left|\mu_{\sigma^{\prime}}\right|}{\ln \left|\mu_{\sigma}\right|}$, where $\mu_{\sigma}$ is the eigenvalue of the map $J_{\sigma}\left(\mu_{\sigma^{\prime}}\right.$ is the eigenvalue of $\left.J_{\sigma^{\prime}}\right)$ that is greater than 1 in absolute value. We define a homeomorphism $\varphi_{\sigma}^{u}: W_{\sigma}^{u} \rightarrow W_{\sigma^{\prime}}^{u}$ by the formula $\varphi_{\sigma}^{u}(w)=w^{\prime}$, where $\left|w_{3}^{\prime}\right|=\left|w_{3}\right|^{r}$, and by the following condition: if $w$ belongs to a connected component $E$ of the set $U_{\sigma} \backslash W_{\sigma}^{s}$, then $w^{\prime}$ belongs to the connected component $E^{\prime}$ of the set $U_{\sigma^{\prime}} \backslash W_{\sigma^{\prime}}^{s}$ such that $\varphi\left(E \backslash W_{\sigma}^{u}\right) \cap E^{\prime} \neq \varnothing$.

It can be verified directly that the homeomorphism $\varphi_{\sigma}^{u}$ conjugates the diffeomorphisms $\left.f^{m_{\sigma}}\right|_{W_{\sigma}^{u}}$ and $\left.f^{\prime m_{\sigma^{\prime}}}\right|_{W_{\sigma^{\prime}}^{u}}$ (see the proof of a similar fact in Step 2 of the proof of Theorem 1 
in [14], for example). Since $\widehat{\varphi}\left(\widehat{W}_{\sigma}^{s}\right)=\widehat{W}_{\sigma^{\prime}}^{s}$ and $\eta_{f}=\eta_{f^{\prime}} \widehat{\varphi}_{*}$, we find that the inclusions $j_{\widehat{W}_{\sigma}^{s}}: \widehat{W}_{\sigma}^{s} \rightarrow \widehat{V}_{f}$ and $j_{\widehat{W}_{\sigma^{\prime}}^{s}}^{\prime}: \widehat{W}_{\sigma^{\prime}}^{s} \rightarrow \widehat{V}_{f^{\prime}}$ satisfy

$$
\eta_{f}\left(j_{\widehat{W}_{\sigma}^{s}} *\left(\pi_{1}\left(\widehat{W}_{\sigma}^{s}\right)\right)\right)=\eta_{f^{\prime}}\left(j_{\widehat{W}_{\sigma^{\prime}}^{\prime} *}^{\prime}\left(\pi_{1}\left(\widehat{W}_{\sigma^{\prime}}^{s}\right)\right)\right) .
$$

On the other hand,

$$
\eta_{f}\left(j_{\widehat{W}_{\sigma}^{s}}\left(\pi_{1}\left(\widehat{W}_{\sigma}^{s}\right)\right)\right)=m_{\sigma} \mathbb{Z} \quad \text { and } \quad \eta_{f^{\prime}}\left(j_{\widehat{W}_{\sigma^{\prime}}^{s}}^{\prime}\left(\pi_{1}\left(\widehat{W}_{\sigma^{\prime}}^{s}\right)\right)\right)=m_{\sigma^{\prime}} \mathbb{Z}
$$

and so $m_{\sigma}=m_{\sigma^{\prime}}$. Then the homeomorphism

$$
\varphi_{f^{k}(\sigma)}^{u}=f^{\prime k} \varphi_{\sigma}^{u} f^{-k}: W_{f^{k}(\sigma)}^{u} \rightarrow W_{f^{\prime k}\left(\sigma^{\prime}\right)}^{u}
$$

conjugates the diffeomorphisms $\left.f^{m_{\sigma}}\right|_{W_{f^{k}(\sigma)}^{u}}$ and $\left.f^{\prime f_{\sigma^{\prime}}^{\prime m}}\right|_{W_{f^{\prime k}\left(\sigma^{\prime}\right)}^{u}}$ for every $k=0, \ldots, m_{\sigma}$.

By performing similar constructions for every periodic orbit of the set $\Omega_{1}$, we obtain the desired homeomorphism $\varphi^{u}: W_{\Omega_{1}}^{u} \rightarrow W_{\Omega_{1}^{\prime}}^{u}$.

Step 3. We define a conjugating homeomorphism $\varphi^{s}: W_{\Omega_{2}}^{s} \rightarrow W_{\Omega_{2}^{\prime}}^{s}$.

Let $\sigma \in \Omega_{2}, \sigma^{\prime}=\varphi(\sigma)$ and let $\psi_{\sigma}, \psi_{\sigma^{\prime}}$ be linearizing diffeomorphisms (see Definition 2.1). We set $\beta=1$ if $W_{\sigma}^{u}$ does not contain heteroclinic tangency points, and define $\beta$ as follows if there is a heteroclinic tangency point $a \in W_{\sigma}^{u}$. We set

$$
g_{a}=\psi_{\sigma_{a}^{u}}\left(\left.\psi_{\sigma_{a}^{s}}\right|_{U_{a}}\right)^{-1}: \psi_{\sigma_{a}^{s}}\left(U_{a}\right) \rightarrow \psi_{\sigma_{a}^{u}}\left(U_{a}\right)
$$

and write down the map $g_{a}$ in coordinate form:

$$
g_{a}\left(x_{1}, x_{2}, x_{3}\right)=\left(\xi_{a}\left(x_{1}, x_{2}, x_{3}\right), \eta_{a}\left(x_{1}, x_{2}, x_{3}\right), \zeta_{a}\left(x_{1}, x_{2}, x_{3}\right)\right) .
$$

We set $\rho=\frac{\ln \left|\lambda_{\sigma^{\prime}}\right|}{\ln \left|\lambda_{\sigma}\right|}$, where $\lambda_{\sigma}$ is the eigenvalue of the map $J_{\sigma}$ and $\lambda_{\sigma^{\prime}}$ is the eigenvalue of $J_{\sigma^{\prime}}$ that is less than 1 in absolute value. We set

$$
\beta=\frac{\frac{\partial \zeta_{a}^{\prime}}{\partial x_{3}}\left(a^{\prime}\right)}{\left(\frac{\partial \zeta_{a}}{\partial x_{3}}(a)\right)^{\rho}}, \quad \text { where } a^{\prime}=\varphi(a) .
$$

For any point $w \in W_{\sigma}^{s}\left(w^{\prime} \in W_{\sigma^{\prime}}^{s}\right)$ the point $\psi_{\sigma}(w)\left(\psi_{\sigma^{\prime}}\left(w^{\prime}\right)\right)$ has coordinates $\left(0,0, w_{3}\right)$ $\left(\left(0,0, w_{3}^{\prime}\right)\right)$. We define a homeomorphism $\varphi_{\sigma}^{s}: W_{\sigma}^{s} \rightarrow W_{\sigma^{\prime}}^{s}$ by the formula $\varphi_{\sigma}^{s}(w)=w^{\prime}$, where $\left|w_{3}^{\prime}\right|=|\beta| \cdot\left|w_{3}\right|^{\rho}$, and by the following condition: if $w$ belongs to a connected component $E$ of the set $U_{\sigma} \backslash W_{\sigma}^{u}$, then $w^{\prime}$ belongs to the connected component $E^{\prime}$ of the set $U_{\sigma^{\prime}} \backslash W_{\sigma^{\prime}}^{u}$ such that $\varphi\left(E \backslash W_{\sigma}^{s}\right) \cap E^{\prime} \neq \varnothing$. It can be verified directly that the homeomorphism

$$
\varphi_{f^{k}(\sigma)}^{s}=f^{\prime k} \varphi_{\sigma}^{s} f^{-k}: W_{f^{k}(\sigma)}^{s} \rightarrow W_{f^{\prime k}\left(\sigma^{\prime}\right)}^{s}
$$

conjugates the diffeomorphisms $\left.f^{m_{\sigma}}\right|_{W_{f^{k}(\sigma)}^{s}}$ and $\left.f^{\prime m_{\sigma^{\prime}}}\right|_{W_{f^{\prime k}\left(\sigma^{\prime}\right)}^{s}}$ for every $k=0, \ldots, m_{\sigma}$ (see the proof of a similar fact in Step 3 of the proof of Theorem 1 in [14, for example).

By performing similar constructions for every periodic orbit of the set $\Omega_{2}$, we obtain a sought-for homeomorphism $\varphi^{s}: W_{\Omega_{2}}^{s} \rightarrow W_{\Omega_{2}^{\prime}}^{s}$.

Step 4 . We modify the homeomorphism $\varphi$ in a neighbourhood of the set $\mathscr{A}$.

To do this we set $a_{l}^{\prime}=\varphi\left(a_{l}\right)$. Then, on some arc $\tilde{\ell}_{a_{l}} \subset \ell_{a_{l}}$ containing the point $a_{l}$, there is a homeomorphism onto the image $\varphi_{\ell_{a_{l}}}: \tilde{\ell}_{a_{l}} \rightarrow \ell_{a_{l}^{\prime}}$ defined by the formula $\varphi_{\ell_{a_{l}}}(z)=z^{\prime}$, where we associate the point $z^{\prime}=F_{\sigma_{a_{l}^{\prime}}^{s}, \varphi^{u}(y)}^{2} \cap \ell_{a_{l}}$ with a point $z \in \tilde{\ell}_{a_{l}}$ that is the point 
of intersection of the leaf $F_{\sigma_{a_{l}}^{s}}^{2}, y, y \in W_{\sigma_{a_{l}}^{s}}^{u}$, and the arc $\tilde{\ell}_{a_{l}}$. We choose a smooth twodimensional disc $d_{l} \subset\left(U_{a_{l}} \cap W_{\sigma_{a_{l}}^{s}}^{s}\right)\left(d_{l}^{\prime} \subset\left(U_{a_{l}^{\prime}} \cap W_{\sigma_{a_{l}^{\prime}}^{s}}^{s}\right)\right)$ containing the point $a_{l}\left(a_{l}^{\prime}\right)$ such that its boundary $c_{l}=\partial d_{l}\left(c_{l}^{\prime}=\partial d_{l}^{\prime}\right)$ intersects every leaf of the foliation $B_{a_{l}}\left(B_{a_{l}^{\prime}}\right)$. We choose an orientation on the curve $c_{l}\left(c_{l}^{\prime}\right)$ which corresponds to moving around in such a way that the disc $d_{l}\left(d_{l}^{\prime}\right)$ remains on the left and denote a homeomorphism preserving the chosen orientation by $\varphi_{c_{l}}: c_{l} \rightarrow c_{l}^{\prime}$. We let $B_{a_{l}, x}\left(B_{a_{l}^{\prime}, x^{\prime}}\right)$ denote the leaf of the foliation $B_{a_{l}}\left(B_{a_{l}^{\prime}}\right)$ passing through the point $x \in c_{l}\left(x^{\prime} \in c_{l}^{\prime}\right)$.

We observe that for any point $z\left(z^{\prime}\right)$ in some neighbourhood of the point $a_{l}\left(a_{l}^{\prime}\right)$ there exists a unique triple of points $\left(z_{1}, z_{2}, z_{3}\right)\left(\left(z_{1}^{\prime}, z_{2}^{\prime}, z_{3}^{\prime}\right)\right)$ such that

$$
z_{1}, z_{2} \in \ell_{a_{l}} \quad\left(z_{1}^{\prime}, z_{2}^{\prime} \in \ell_{a_{l}^{\prime}}\right), \quad z_{3} \in c_{l} \quad\left(z_{3}^{\prime} \in c_{l}^{\prime}\right),
$$

and

$$
z=F_{\sigma_{a_{l}}^{s}, z_{1}}^{2} \cap F_{\sigma_{a_{l}}^{u}, z_{2}}^{2} \cap B_{a_{l}, z_{3}} \quad\left(z^{\prime}=F_{\sigma_{a_{l}^{\prime}}^{s}, z_{1}^{\prime}}^{2} \cap F_{\sigma_{a_{l}^{\prime}}^{u}, z_{2}^{\prime}}^{2} \cap B_{a_{l}^{\prime}, z_{3}^{\prime}}\right) .
$$

Then in some neighbourhood of the point $a_{l}$, a homeomorphism onto the image $\varphi_{a_{l}}$ is well defined that associates with a point $z$ a point $z^{\prime}$ such that $z_{1}^{\prime}=\varphi_{\ell_{a_{l}}}\left(z_{1}\right), z_{2}^{\prime}=\varphi_{\ell_{a_{l}}}\left(z_{2}\right)$, $z_{3}^{\prime}=\varphi_{c_{l}}\left(z_{3}\right)$. We choose 3-balls $V_{a_{l}}^{1}, V_{a_{l}}^{2}$ with the following properties:

1) $V_{a_{l}}^{1} \subset \operatorname{int} V_{a_{l}}^{2}$ and $f^{k}\left(V_{a_{l}}^{2}\right) \cap V_{a_{l}}^{2}=\varnothing$ for any $k \in(\mathbb{Z} \backslash\{0\})$;

2) the spheres $\Sigma_{a_{l}}^{1}=\partial V_{a_{l}}^{1}$ and $\Sigma_{a_{l}}^{2}=\partial V_{a_{l}}^{2}$ intersect every manifold $W_{\sigma_{a_{l}}^{s}}^{s}$ and $W_{\sigma_{a_{l}}^{u}}^{u}$ in one circle;

3) there is a well-defined homeomorphism $\varphi_{a_{l}}$ on the set $V_{a_{l}}^{1}$ and $\varphi_{a_{l}}\left(V_{a_{l}}^{1}\right) \subset \operatorname{int} \varphi\left(V_{a_{l}}^{2}\right)$.

We construct a homeomorphism onto the image $\varphi_{V_{a_{l}}^{2}}$ that coincides with $\varphi_{a_{l}}$ on $V_{a_{l}}^{1}$, coincides with $\varphi$ on $\Sigma^{2}$, and is such that $\varphi_{V_{a_{l}}^{2}}\left(W_{\sigma_{a_{l}}^{s}}^{s} \cap V_{a_{l}}^{2}\right) \subset W_{\sigma_{a_{l}^{\prime}}^{s}}^{s}$ and $\varphi_{V_{a_{l}}^{2}}\left(W_{\sigma_{a_{l}}^{u}}^{u} \cap V_{a_{l}}^{2}\right) \subset$ $W_{\sigma_{l}^{\prime}}^{u}$. To do this it is sufficient to construct a homeomorphism $\phi_{V_{a_{l}}^{2}}: V_{a_{l}}^{2} \rightarrow V_{a_{l}}^{2}$ that coincides with $\phi_{V_{a_{l}}^{1}}=\varphi^{-1} \varphi_{a_{l}}$ on $V_{a_{l}}^{1}$, coincides with id on $\Sigma^{2}$, and satisfies the conditions $\phi_{V_{a_{l}}^{2}}\left(W_{\sigma_{a_{l}}^{s}}^{s} \cap V_{a_{l}}^{2}\right)=W_{\sigma_{a_{l}}^{s}}^{s} \cap V_{a_{l}}^{2}$ and $\varphi_{V_{a_{l}}^{2}}\left(W_{\sigma_{a_{l}}^{u}}^{u} \cap V_{a_{l}}^{2}\right)=W_{\sigma_{a_{l}}^{u}}^{u} \cap V_{a_{l}}^{2}$. Then $\varphi_{V_{a_{l}}^{2}}=\varphi \phi_{V_{a_{l}}^{2}}$ is the required map.

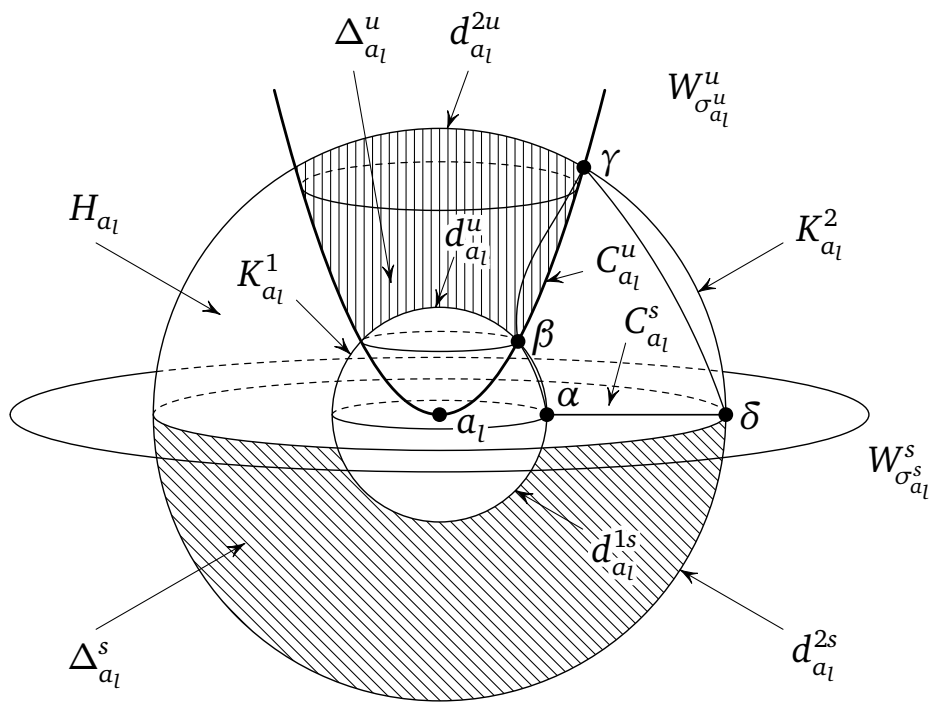

Figure 6. Three-dimensional annulus $G_{a_{l}}$ 
We set $\widetilde{V}_{a_{l}}^{1}=\phi_{V_{a_{l}}^{1}}\left(V_{a_{l}}^{1}\right), \widetilde{\Sigma}_{a_{l}}^{1}=\partial \widetilde{V}_{a_{l}}^{1}, G_{a_{l}}=c l\left(V_{a_{l}}^{2} \backslash V_{a_{l}}^{1}\right)$ and $\widetilde{G}_{a_{l}}=c l\left(V_{a_{l}}^{2} \backslash \widetilde{V}_{a_{l}}^{1}\right)$. We observe that the set $G_{a_{l}}\left(\widetilde{G}_{a_{l}}\right)$ is a three-dimensional annulus and the two-dimensional cylinders $C_{a_{l}}^{u}=G_{a_{l}} \cap W_{\sigma_{a_{l}}^{u}}^{u}$ and $C_{a_{l}}^{s}=G_{a_{l}} \cap W_{\sigma_{a_{l}}^{u}}^{s}\left(\widetilde{C}_{a_{l}}^{u}=\widetilde{G}_{a_{l}} \cap W_{\sigma_{a_{l}}^{u}}^{u}\right.$ and $\widetilde{C}_{a_{l}}^{s}=\widetilde{G}_{a_{l}} \cap W_{\sigma_{a_{l}}^{s}}^{s}$, respectively) divide it into three parts, $\Delta_{a_{l}}^{u}, \Delta_{a_{l}}^{s}, H_{a_{l}}\left(\widetilde{\Delta}_{a_{l}}^{u}, \widetilde{\Delta}_{a_{l}}^{s}, \widetilde{H}_{a_{l}}\right)$. Here $\Delta_{a_{l}}^{u}$, which is the set with the vertical hatching in Figure [6 is the three-dimensional ball bounded by the sphere $S_{a_{l}}^{u}$ composed of the annulus $C_{a_{l}}^{u}$ and the discs $d_{a_{l}}^{1 u} \subset \Sigma_{a_{l}}^{1}, d_{a_{l}}^{2 u} \subset \Sigma_{a_{l}}^{2}\left(\widetilde{\Delta}_{a_{l}}^{u}\right.$ is the three-dimensional ball bounded by the sphere $\widetilde{S}_{a_{l}}^{u}$ composed of the annulus $\widetilde{C}_{a_{l}}^{u}$ and the $\left.\operatorname{discs} \widetilde{d}_{a_{l}}^{1 u} \subset \widetilde{\Sigma}_{a_{l}}^{1}, d_{a_{l}}^{2 u} \subset \Sigma_{a_{l}}^{2}\right) ; \Delta_{a_{l}}^{s}$, which is the set with the slanting hatching in Figure 6. is the three-dimensional ball bounded by the sphere $S_{a_{l}}^{s}$ composed of the annulus $C_{a_{l}}^{s}$ and the discs $d_{a_{l}}^{1 s} \subset \Sigma_{a_{l}}^{1}$ and $d_{a_{l}}^{2 s} \subset \Sigma_{a_{l}}^{2}\left(\widetilde{\Delta}_{a_{l}}^{s}\right.$ is the three-dimensional ball bounded by the sphere $\widetilde{S}_{a_{l}}^{s}$ composed of the annulus $\widetilde{C}_{a_{l}}^{s}$ and the discs $\left.\widetilde{d}_{a_{l}}^{1 s} \subset \widetilde{\Sigma}_{a_{l}}^{1}, d_{a_{l}}^{2 s} \subset \Sigma_{a_{l}}^{2}\right) ; H_{a_{l}}$ is the solid torus bounded by the torus $T_{a_{l}}$ composed of the cylinders $C_{a_{l}}^{u}, C_{a_{l}}^{s}$ and the annuli $k_{a_{l}}^{1} \subset \Sigma_{a_{l}}^{1}$ and $k_{a_{l}}^{2} \subset \Sigma_{a_{l}}^{2}\left(\widetilde{H}_{a_{l}}\right.$ is the solid torus bounded by the torus $\widetilde{T}_{a_{l}}$ composed of the cylinders $\widetilde{C}_{a_{l}}^{u}, \widetilde{C}_{a_{l}}^{s}$ and the annuli $\widetilde{k}_{a_{l}}^{1} \subset \Sigma_{a_{l}}^{1}$ and $\left.k_{a_{l}}^{2} \subset \Sigma_{a_{l}}^{2}\right)$; see Figure 6 ,

By construction, $\widetilde{d}_{a_{l}}^{1 s}=\phi_{V_{a_{l}}^{1}}\left(d_{a_{l}}^{1 s}\right), \widetilde{d}_{a_{l}}^{1 u}=\phi_{V_{a_{l}}^{1}}\left(d_{a_{l}}^{1 u}\right)$ and $\widetilde{k}_{a_{l}}^{1}=\phi_{V_{a_{l}}^{1}}\left(k_{a_{l}}^{1}\right)$. The choice of the homeomorphism $\varphi_{c_{l}}$ implies that there exists a homeomorphism $\phi_{C_{a_{l}}^{u}}^{u}: C_{a_{l}}^{u} \rightarrow \widetilde{C}_{a_{l}}^{u}$ coinciding with $\phi_{V_{a_{l}}^{1}}$ on $C_{a_{l}}^{u} \cap \Sigma_{a_{l}}^{1}$ and equal to the identity on $C_{a_{l}}^{u} \cap \Sigma_{a_{l}}^{2}$. Then on the sphere $S_{a_{l}}^{u}$ we have constructed a homeomorphism $\phi_{S_{a_{l}}^{u}}: S_{a_{l}}^{u} \rightarrow \widetilde{S}_{a_{l}}^{u}$ coinciding with $\phi_{C_{a_{l}}^{u}}$ on $C_{a_{l}}^{u}$, with $\phi_{V_{a_{l}}^{1}}$ on $d_{a_{l}}^{1 u}$ and equal to the identity on $d_{a_{l}}^{2 u}$. Let $\phi_{\Delta_{a_{l}}^{u}}: D_{a_{l}}^{u} \rightarrow \widetilde{\Delta}_{a_{l}}^{u}$ denote an extension of the homeomorphism $\phi_{S_{a_{l}}^{u}}$ to the ball $\Delta_{a_{l}}^{u}$. We choose a meridian $c \subset T_{a_{l}}$ of the solid torus $H_{a_{l}}$ (that is, a closed curve contractible on $H_{a_{l}}$ but not contractible on $\left.T_{a_{l}}\right)$ in such a way that it consists of closed $\operatorname{arcs}[\alpha, \beta] \subset \Sigma_{a_{l}}^{1},[\beta, \gamma] \subset C_{a_{l}}^{u},[\gamma, \delta] \subset \Sigma_{a_{l}}^{2}$ and $[\delta, \alpha] \subset C_{a_{l}}^{s}$ (see Figure [6). We set $[\widetilde{\alpha}, \widetilde{\beta}]=\phi_{V_{a_{l}}^{1}}([\alpha, \beta])$ and $[\widetilde{\beta}, \gamma]=\phi_{C_{a_{l}}^{u}}([\beta, \gamma])$. We choose a curve $[\delta, \widetilde{\alpha}] \subset \widetilde{C}_{a_{l}}^{s}$ such that the closed curve $\tilde{c} \subset \widetilde{T}_{a_{l}}$ composed of the closed arcs $[\widetilde{\alpha}, \widetilde{\beta}] \subset \widetilde{\Sigma}_{a_{l}}^{1},[\widetilde{\beta}, \gamma] \subset \widetilde{C}_{a_{l}}^{u},[\gamma, \delta] \subset \Sigma_{a_{l}}^{2}$ and $[\delta, \widetilde{\alpha}] \subset \widetilde{C}_{a_{l}}^{s}$ is a meridian of the solid torus $\widetilde{H}_{a_{l}}$. Since $\widetilde{C}_{a_{l}}^{s} \backslash[\delta, \widetilde{\alpha}]$ is a two-dimensional disc, the choice of the homeomorphism $\varphi_{c_{l}}$ implies that there exists a homeomorphism $\phi_{C_{a_{l}}^{s}}: C_{a_{l}}^{s} \rightarrow \widetilde{C}_{a_{l}}^{s}$ that coincides with $\phi_{V_{a_{l}}^{1}}$ on $C_{a_{l}}^{s} \cap \Sigma_{a_{l}}^{1}$, is equal to the identity on $C_{a_{l}}^{s} \cap \Sigma_{a_{l}}^{2}$, and is such that $\phi_{C_{a_{l}}^{s}}([\delta, \alpha])=[\delta, \widetilde{\alpha}]$. Then on the torus $T_{a_{l}}$ we have constructed a homeomorphism $\phi_{T_{a_{l}}}: T_{a_{l}} \rightarrow \widetilde{T}_{a_{l}}$ that coincides with $\phi_{C_{a_{l}}^{u}}$ on $C_{a_{l}}^{u}$, with $\phi_{C_{a_{l}}^{s}}$ on $C_{a_{l}}^{s}$, with $\phi_{V_{a_{l}}^{1}}$ on $k_{a_{l}}^{1}$, and is equal to the identity on $k_{a_{l}}^{2}$. Since the map $\phi_{T_{a_{l}}}$ takes a meridian of the torus $H_{a_{l}}$ to a meridian of the torus $\widetilde{T}_{a_{l}}$, this map can be extended to a homeomorphism $\phi_{H_{a_{l}}}: H_{a_{l}} \rightarrow \widetilde{H}_{a_{l}}$. This automatically constructs a homeomorphism $\phi_{S_{a_{l}}^{s}}: S_{a_{l}}^{s} \rightarrow \widetilde{S}_{a_{l}}^{s}$ on the sphere $S_{a_{l}}^{s}$ that coincides with $\phi_{C_{a_{l}}^{s}}$ on $C_{a_{l}}^{s}$, with $\phi_{V_{a_{l}}^{1}}$ on $d_{a_{l}}^{1 s}$, and is equal to the identity on $d_{a_{l}}^{2 s}$. Let $\phi_{\Delta_{a_{l}}^{s}}: \Delta_{a_{l}}^{s} \rightarrow \widetilde{\Delta}_{a_{l}}^{s}$ denote an extension of the homeomorphism $\phi_{S_{a_{l}}^{s}}$ to the ball $\Delta_{a_{l}}^{s}$. Finally, the homeomorphism we require, $\phi_{V_{a_{l}}^{2}}: V_{a_{l}}^{2} \rightarrow V_{a_{l}}^{2}$, coincides with $\phi_{V_{a_{l}}^{1}}$ on $V_{a_{l}}^{1}$, with $\phi_{\Delta_{a_{l}}^{s}}$ on $\Delta_{a_{l}}^{s}$, with $\phi_{H_{a_{l}}}$ on $H_{a_{l}}$ and with $\phi_{\Delta_{a_{l}}^{u}}$ on $\Delta_{a_{l}}^{u}$.

We set $V_{\mathscr{A}}^{2}=V_{a_{1}}^{2} \cup \cdots \cup V_{a_{k}}^{2}$ and let $\varphi_{V_{\mathscr{A}}^{2}}$ denote the homeomorphism composed of the homeomorphisms $\varphi \phi_{V_{a_{1}}^{2}}, \ldots, \varphi \phi_{V_{a_{k}}^{2}}$. Let $\varphi_{0}: V_{f} \rightarrow V_{f^{\prime}}$ denote the homeomorphism that coincides with $f^{\prime n} \varphi_{V_{\mathscr{A}}^{2}} f^{-n}$ on $f^{n}\left(V_{\mathscr{A}}^{2}\right), n \in \mathbb{Z}$, and coincides with $\varphi$ outside $\bigcup_{n \in \mathbb{Z}} f^{n}\left(V_{\mathscr{A}}^{2}\right)$. 
We observe that for any point $y\left(y^{\prime}\right)$ in the set $V_{a_{l}}^{1} \backslash a_{l}\left(\varphi_{0}\left(V_{a_{l}}^{1}\right) \backslash a_{l}^{\prime}\right)$ there exists a unique pair of points $\left(y_{s}, y_{u}\right)\left(\left(y_{s}^{\prime}, y_{u}^{\prime}\right)\right)$ such that

$$
y_{s} \in W_{\sigma_{a_{l}}^{s}}^{s}, \quad y_{u} \in W_{\sigma_{a_{l}}^{s}}^{u} \quad\left(y_{s}^{\prime} \in W_{\sigma_{a_{l}^{\prime}}^{s}}^{s}, \quad y_{u}^{\prime} \in W_{\sigma_{a_{l}^{\prime}}^{s}}^{u}\right)
$$

and

$$
y=F_{\sigma_{a_{l}}^{s}, y_{u}}^{2} \cap F_{\sigma_{a_{l}}^{s}, y_{s}}^{1} \quad\left(y^{\prime}=F_{\sigma_{l}^{\prime}}^{2}, y_{u}^{\prime} \cap F_{\sigma_{l}^{\prime}}^{1}, y_{s}^{\prime}\right) .
$$

Then $\varphi_{0}(y)=y^{\prime}$, where $y_{s}^{\prime}=\varphi_{0}\left(y_{s}\right)$ and $y_{u}^{\prime}=\varphi^{u}\left(y_{u}\right)$. At the same time, there exists a unique pair of points $\left(\widetilde{y}_{s}, \widetilde{y}_{u}\right)$ for the point $y\left(\left(\widetilde{y}_{s}^{\prime}, \widetilde{y}_{u}^{\prime}\right)\right.$ for $\left.y^{\prime}\right)$ such that

$$
\widetilde{y}_{s} \in W_{\sigma_{a_{l}}^{u}}^{s}, \quad \widetilde{y}_{u} \in W_{\sigma_{a_{l}}^{u}}^{u} \quad\left(\widetilde{y}_{s}^{\prime} \in W_{\sigma_{a_{l}^{\prime}}^{u}}^{s}, \quad \widetilde{y}_{u}^{\prime} \in W_{\sigma_{a_{l}^{\prime}}^{u}}^{u}\right)
$$

and

$$
y=F_{\sigma_{a_{l}}^{u}, \widetilde{y}_{s}}^{2} \cap F_{\sigma_{a_{l}}^{u}}^{1}, \widetilde{y}_{u} \quad\left(y^{\prime}=F_{\sigma_{a_{l}^{\prime}}^{u}, \widetilde{y}_{s}^{\prime}}^{2} \cap F_{\sigma_{a_{l}^{\prime}}^{u}, \widetilde{y}_{u}^{\prime}}^{1}\right) .
$$

Then $\varphi_{0}(y)=y^{\prime}$, where $\widetilde{y}_{s}^{\prime}=\varphi^{s}\left(\widetilde{y}_{s}\right)$ and $\widetilde{y}_{u}^{\prime}=\varphi_{0}\left(\widetilde{y}_{u}\right)$.

Step 5. We modify the homeomorphism $\varphi_{1}$ on the set $U_{\Omega_{1}}=\bigcup_{\sigma \in \Omega_{1}} U_{\sigma}$.

By construction, for every curve $\ell_{a_{l}} \cap V_{a_{l}}^{1}$ there exists a neighbourhood $N_{a_{l}}^{u} \subset V_{a_{l}}^{1}$ composed of one-dimensional compatible leaves of the diffeomorphism $f$. Property 5) in Definition 3.1 and the construction of the homeomorphism $\varphi_{0}$ imply that the set $N_{a_{l}^{\prime}}^{u}=\varphi_{0}\left(N_{a_{l}}^{u}\right)$ is a neighbourhood of the curve $\ell_{a_{l}^{\prime}} \cap \varphi_{0}\left(V_{a_{l}}^{1}\right)$ composed of one-dimensional compatible leaves of the diffeomorphism $f^{\prime}$. We set $N_{\mathscr{A}}^{u}=N_{a_{1}}^{u} \cup \cdots \cup N_{a_{k}}^{u}$ and $N_{\mathscr{A}^{\prime}}^{u}=$ $N_{a_{1}^{\prime}}^{u} \cup \cdots \cup N_{a_{k}^{\prime}}^{u}$.

Let $\sigma \in \Omega_{1}, \sigma^{\prime}=\varphi(\sigma)$ and let $U_{\sigma}, U_{\sigma^{\prime}}$ be neighbourhoods in a compatible system. We set $N_{\sigma}^{u}=U_{\sigma} \cap \bigcup_{n \in \mathbb{Z}} f^{n}\left(N_{\mathscr{A}}^{u}\right)\left(N_{\sigma^{\prime}}^{u}=U_{\sigma^{\prime}} \cap \bigcup_{n \in \mathbb{Z}} f^{n}\left(N_{\mathscr{A}^{\prime}}^{u}\right)\right)$. We observe that for any point $y\left(y^{\prime}\right)$ in the set $\widetilde{U}_{\sigma}=U_{\sigma} \backslash N_{\sigma}^{u}\left(\widetilde{U}_{\sigma^{\prime}}=U_{\sigma^{\prime}} \backslash N_{\sigma^{\prime}}^{u}\right)$ there exists a unique pair of points $\left(y_{s}, y_{u}\right)$ such that $y_{s} \in W_{\sigma}^{s}$ and $y_{u} \in W_{\sigma}^{u}$ and $y=F_{\sigma, y_{s}}^{1} \cap F_{\sigma, y_{u}}^{2}\left(\left(y_{s}^{\prime}, y_{u}^{\prime}\right)\right.$ such that $y_{s}^{\prime} \in W_{\sigma^{\prime}}^{s}$ and $y_{u}^{\prime} \in W_{\sigma^{\prime}}^{u}$ and $\left.y^{\prime}=F_{\sigma^{\prime}, y_{s}^{\prime}}^{1} \cap F_{\sigma^{\prime}, y_{u}^{\prime}}^{2}\right)$. Then there exists an $f^{m_{\sigma_{-}}}$ invariant neighbourhood $V_{\sigma} \subset U_{\sigma}$ of the point $\sigma$ such that on the set $\widetilde{V}_{\sigma}=V_{\sigma} \backslash N_{\sigma}^{u}$ a homeomorphism onto the image $\varphi_{\tilde{V}_{\sigma}}: \widetilde{V}_{\sigma} \rightarrow U_{\sigma^{\prime}}$ is well defined that associates with a point $y \in \widetilde{V}_{\sigma}$ a point $y^{\prime}$ such that $y_{s}^{\prime}=\varphi_{0}\left(y_{s}\right)$ and $y_{u}^{\prime}=\varphi^{u}\left(y_{u}\right)$. We assume without loss of generality that the set $V_{\sigma}$ is chosen in such a way that $\varphi_{\tilde{V}_{\sigma}}\left(\widetilde{V}_{\sigma}\right) \subset \varphi_{0}\left(\widetilde{U}_{\sigma}\right)$.

We define a topological embedding $\phi_{\sigma}: \widetilde{V}_{\sigma} \backslash W_{\sigma}^{u} \rightarrow \widetilde{U}_{\sigma}$ by the formula $\phi_{\sigma}=\varphi_{0}^{-1} \varphi_{\tilde{V}_{\sigma}}$. We set $Z_{\sigma}=U_{\sigma} \cap W_{\Omega_{2}}^{u}$. The properties of a compatible system of neighbourhoods imply that the set $Z_{\sigma}$ consists of one-dimensional leaves of the foliation $F_{\sigma}^{1}$. By construction, the topological embedding $\phi_{\sigma}$ is the identity on the set $\partial N_{\sigma}^{u}$ and $\phi_{\sigma}\left(Z_{\sigma}\right) \subset W_{\Omega_{2}}^{u}$. Then, by Lemma 4.3.2 and Corollary 4.3.2 in the book [8], there exists a homeomorphism $\Phi_{\sigma}: U_{\sigma} \backslash W_{\sigma}^{u} \rightarrow U_{\sigma} \backslash W_{\sigma}^{u}$ commuting with the diffeomorphism $\left.f^{m_{\sigma}}\right|_{U_{\sigma} \backslash W_{\sigma}^{u}}$, coinciding with $\phi_{\sigma}$ on $\widetilde{V}_{\sigma} \backslash W_{\sigma}^{u}$, equal to the identity on $N_{\sigma}^{u} \cup \partial U_{\sigma}$, and such that $\Phi_{\sigma}\left(U_{\sigma} \cap W_{\Omega_{2}}^{u}\right)=U_{\sigma} \cap W_{\Omega_{2}}^{u}$. We define a topological embedding $\varphi_{\sigma}: U_{\sigma} \backslash W_{\sigma}^{u} \rightarrow U_{\sigma^{\prime}} \backslash W_{\sigma^{\prime}}^{u}$ by the formula $\varphi_{\sigma}=\varphi_{0} \phi_{\sigma}$. By construction, the homeomorphism $\varphi_{\sigma}$ can be continuously extended to $W_{\sigma}^{u}$ by a homeomorphism $\varphi_{\sigma}^{u}$.

For every $k=0, \ldots, m_{\sigma}$ we define a homeomorphism onto the image by the formula $\varphi_{f^{k}(\sigma)}=f^{\prime k} \varphi_{\sigma} f^{-k}: U_{f^{k}(\sigma)} \backslash W_{f^{k}(\sigma)}^{u} \rightarrow U_{f^{\prime k}\left(\sigma^{\prime}\right)} \backslash W_{f^{\prime k}\left(\sigma^{\prime}\right)}^{u}$. Let $\varphi_{\Omega_{1}}$ denote the map composed of $\varphi_{\sigma}, \sigma \in \Omega_{1}$. Then the desired homeomorphism $\varphi_{1}: V_{f} \rightarrow V_{f^{\prime}}$ coincides with $\varphi_{\Omega_{1}}$ on $U_{\Omega_{1}} \backslash W_{\Omega_{1}}^{u}$ and with $\varphi_{0}$ outside $U_{\Omega_{1}}$. 
Step 6. We modify the homeomorphism $\varphi_{1}$ on the set $U_{\Omega_{2}}=\bigcup_{\sigma \in \Omega_{2}} U_{\sigma}$. The equation $\Theta_{a_{l}}=\Theta_{a_{l}^{\prime}}$ for $a_{l} \in \mathscr{A}$ implies the relation

$$
\frac{\ln \left|\mu_{\sigma_{a_{l}^{\prime}}^{s}}\right|}{\ln \left|\mu_{\sigma_{a_{l}}^{s}}\right|}=\frac{\ln \left|\lambda_{\sigma_{a_{l}^{\prime}}^{u}}\right|}{\ln \left|\lambda_{\sigma_{a_{l}}^{u}}\right|} .
$$

Then given a point $z \in \ell_{a_{l}}$ that is the intersection point of the leaf $F_{\sigma_{a_{l}}^{u}, y}^{2}, y \in W_{\sigma_{a_{l}}^{u}}^{s}$ and the arc $\ell_{a_{l}}$, the map $\varphi_{\ell_{a_{l}}}: \ell_{a_{l}} \rightarrow \ell_{a_{l}^{\prime}}$ associates $z$ with the point $z^{\prime}=F_{\sigma_{a_{l}^{\prime}}^{\prime u}, \varphi^{s}(y)}^{2} \cap \ell_{a_{l}^{\prime}}$ (for a proof of a similar fact see Step 4 of the proof of Theorem 1 in [14]).

By construction, for every curve $\ell_{a_{l}} \cap V_{a_{l}}^{1}$ there exists a neighbourhood $N_{a_{l}}^{s} \subset V_{a_{l}}^{1}$ composed of one-dimensional stable compatible leaves of the diffeomorphism $f$. It follows from property 5) in Definition 3.1 and from the construction of the homeomorphism $\varphi_{1}$ that the set $N_{a_{l}^{\prime}}^{s}=\varphi_{1}\left(N_{a_{l}}^{s}\right)$ is a neighbourhood of the curve $\ell_{a_{l}^{\prime}} \cap \varphi_{1}\left(V_{a_{l}}^{1}\right)$ composed of one-dimensional stable compatible leaves of the diffeomorphism $f^{\prime}$. We set $N_{\mathscr{A}}^{s}=$ $N_{a_{1}}^{s} \cup \cdots \cup N_{s_{k}}^{u}$ and $N_{\mathscr{A}^{\prime}}^{u}=N_{a_{1}^{\prime}}^{s} \cup \cdots \cup N_{a_{k}^{\prime}}^{s}$.

Let $\sigma \in \Omega_{2}, \sigma^{\prime}=\varphi(\sigma)$, and let $U_{\sigma}, U_{\sigma^{\prime}}$ be neighbourhoods in a compatible system. We set $N_{\sigma}^{s}=U_{\sigma} \cap \bigcup_{n \in \mathbb{Z}} f^{n}\left(N_{\mathscr{A}}^{s}\right)$ and $N_{\sigma^{\prime}}^{s}=U_{\sigma^{\prime}} \cap \bigcup_{n \in \mathbb{Z}} f^{n}\left(N_{\mathscr{A}^{\prime}}^{s}\right)$. We observe that for any point $y$ in the set $\widetilde{U}_{\sigma}=U_{\sigma} \backslash N_{\sigma}^{s}\left(y^{\prime}\right.$ in $\left.\widetilde{U}_{\sigma^{\prime}}=U_{\sigma^{\prime}} \backslash N_{\sigma^{\prime}}^{s}\right)$ there exists a unique pair of points $\left(y_{s}, y_{u}\right)\left(\left(y_{s}^{\prime}, y_{u}^{\prime}\right)\right)$ such that

$$
y_{s} \in W_{\sigma}^{s}, \quad y_{u} \in W_{\sigma}^{u} \quad\left(y_{s}^{\prime} \in W_{\sigma^{\prime}}^{s}, \quad y_{u}^{\prime} \in W_{\sigma^{\prime}}^{u}\right)
$$

and

$$
y=F_{\sigma, y_{s}}^{2} \cap F_{\sigma, y_{u}}^{1} \quad\left(y^{\prime}=F_{\sigma^{\prime}, y_{s}^{\prime}}^{2} \cap F_{\sigma^{\prime}, y_{u}^{\prime}}^{1} \text {, respectively }\right) .
$$

Then there exists an $f^{m_{\sigma}}$-invariant neighbourhood $V_{\sigma} \subset U_{\sigma}$ of the point $\sigma$ such that a homeomorphism onto the image $\varphi_{V_{\sigma}}: \widetilde{V}_{\sigma} \rightarrow U_{\sigma^{\prime}}$ is well defined on the set $\widetilde{V}_{\sigma}=V_{\sigma} \backslash N_{\sigma}^{s}$ and that given a point $y \in \widetilde{V}_{\sigma}$ it sets up a correspondence between it and the point $y^{\prime}$ such that $y_{u}^{\prime}=\varphi_{1}\left(y_{u}\right)$ and $y_{s}^{\prime}=\varphi^{s}\left(y_{s}\right)$. We assume without loss of generality that the set $V_{\sigma}$ is chosen in such a way that $\varphi_{\tilde{V}_{\sigma}}\left(\widetilde{V}_{\sigma}\right) \subset \varphi_{1}\left(\widetilde{U}_{\sigma}\right)$.

We define a topological embedding $\phi_{\sigma}: \widetilde{V}_{\sigma} \backslash W_{\sigma}^{s} \rightarrow \widetilde{U}_{\sigma}$ by $\phi_{\sigma}=\varphi_{1}^{-1} \varphi_{\tilde{V}_{\sigma}}$. We set $Z_{\sigma}=U_{\sigma} \cap W_{\Omega_{1}}^{s}$. It follows from the properties of a compatible system of neighbourhoods that the set $Z_{\sigma}$ has a neighbourhood $N_{Z_{\sigma}}$ consisting of connected components $K$ of the intersection of two-dimensional stable compatible leaves with $N_{Z_{\sigma}}$ fibred by onedimensional stable leaves of the foliation $F_{\sigma}^{1}$. By construction, the topological embedding $\phi_{\sigma}$ is equal to the identity on the set $\partial N_{\sigma}^{s}$, and $\phi_{\sigma}(K) \subset F_{\widetilde{\sigma}, x}^{2}, \widetilde{\sigma} \in \Omega_{1}$, for a two-dimensional stable leaf $F_{\widetilde{\sigma}, x}^{2}$ such that $K \subset F_{\widetilde{\sigma}, x}^{2}$. Then, by Lemma 4.3.2 and Corollary 4.3.2 in [8], there exists a homeomorphism $\Phi_{\sigma}: U_{\sigma} \backslash W_{\sigma}^{s} \rightarrow U_{\sigma} \backslash W_{\sigma}^{s}$ commuting with the diffeomorphism $\left.f^{m_{\sigma}}\right|_{U_{\sigma} \backslash W_{\sigma}^{u}}$, coinciding with $\phi_{\sigma}$ on $\widetilde{V}_{\sigma} \backslash W_{\sigma}^{s}$, equal to the identity on $N_{\sigma}^{s} \cup \partial U_{\sigma}$, and such that $\Phi_{\sigma}\left(U_{\sigma} \cap K\right)=K$. We define a topological embedding $\varphi_{\sigma}: U_{\sigma} \backslash W_{\sigma}^{s} \rightarrow U_{\sigma^{\prime}} \backslash W_{\sigma^{\prime}}^{s}$ by $\varphi_{\sigma}=\varphi_{1} \phi_{\sigma}$. By construction the homeomorphism $\varphi_{\sigma}$ can be continuously extended to $W_{\sigma}^{s}$ by a homeomorphism $\varphi_{\sigma}^{s}$.

For every $k=0, \ldots, m_{\sigma}$ we define a homeomorphism onto the image by the formula

$$
\varphi_{f^{k}(\sigma)}=f^{\prime k} \varphi_{\sigma} f^{-k}: U_{f^{k}(\sigma)} \backslash W_{f^{k}(\sigma)}^{s} \rightarrow U_{f^{\prime k}\left(\sigma^{\prime}\right)} \backslash W_{f^{\prime k}\left(\sigma^{\prime}\right)}^{s}
$$

Let $\varphi_{\Omega_{2}}$ denote the map composed of $\varphi_{\sigma}, \sigma \in \Omega_{2}$. Then the homeomorphism we require, $\varphi_{2}: V_{f} \rightarrow V_{f^{\prime}}$, coincides with $\varphi_{\Omega_{2}}$ on $U_{\Omega_{2}} \backslash W_{\Omega_{1}}^{s}$ and with $\varphi_{1}$ outside $U_{\Omega_{2}}$.

Finally, we extend the homeomorphism $\varphi_{2}$ to the homeomorphism $h: M^{3} \rightarrow M^{3}$ we are looking for as follows: we set $\left.h\right|_{W_{\Omega_{1}}^{u}}=\left.\varphi^{u}\right|_{W_{\Omega_{1}}^{u}}$ and $\left.h\right|_{W_{\Omega_{2}}^{s}}=\left.\varphi^{s}\right|_{W_{\Omega_{2}}^{s}}$, and for any 
point $\omega \in \Omega_{0}$ we set $h(\omega)=\omega^{\prime} \in \Omega_{0}^{\prime}$ where $\varphi_{3}\left(W_{\omega}^{s} \backslash \omega\right)=W_{\omega^{\prime}}^{s} \backslash \omega^{\prime}$ (for any point $\alpha \in \Omega_{3}$ set $h(\alpha)=\alpha^{\prime} \in \Omega_{3}^{\prime}$, where $\left.\varphi_{3}\left(W_{\alpha}^{u} \backslash \alpha\right)=W_{\alpha^{\prime}}^{u} \backslash \alpha^{\prime}\right)$.

\section{REFERENCES}

[1] A. A. Andronov and L. S. Pontryagin, Rough systems, Dokl. Akad. Nauk SSSR 14 (1937), no. 5, 247-250. (Russian)

[2] A. N. Bezdenezhnykh and V. Z. Grines, Dynamical properties and topological classification of gradient-like diffeomorphisms on two-dimensional manifolds. I, Methods of the qualitative theory of differential equations, Gor'kov. Gos. Univ., Gor'kiı̌, 1984, 22-38; English transl., Selected translations. Selecta Math. Soviet. 11 (1992), no. 1, 1-11. MR866686

[3] A. N. Bezdenezhnykh and V. Z. Grines, Dynamical properties and topological classification of gradient-like diffeomorphisms on two-dimensional manifolds. II, Methods of the qualitative theory of differential equations, Gor'kov. Gos. Univ., Gor'kiř, 1987, 24-31; English transl., Selected translations. Selecta Math. Soviet. 11 (1992), no. 1, 13-17. MR1155895

[4] G. R. Belitskiı̌, Normal forms, invariant and local mappings, Naukova Dumka, Kiev, 1979. (Russian) MR:537763

[5] Ch. Bonatti, V. Grines, V. Medvedev, and E. Pécou, Topological classification of gradient-like diffeomorphisms on 3-manifolds, Topology 43 (2004), no. 2, 369-391. MR2052968

[6] Ch. Bonatti, V. Z. Grines, and O. V. Pochinka, Classification of Morse-Smale diffeomorphisms with a finite set of heteroclinic orbits on 3-manifolds, Trudy Mat. Inst. Steklova 250 (2005), 5-53; English transl., Proc. Steklov Inst. Math. 250 (2005), 1-46. MR2200906

[7] V. Z. Grines and O. V. Pochinka, Morse-Smale cascades on 3-manifolds, Uspekhi Mat. Nauk 68 (2013), no. 1, 129-188; English transl., Russian Math. Surveys 68 (2013), no. 1, 117-173. MR 3088080

[8] V. Z. Grines and O. V. Pochinka, Introduction to the topological classification of cascades on manifolds of dimension two and three, Regular and Chaotic Dynamics, Izhevsk, 2011. (Russian)

[9] V. Z. Grines and O. V. Pochinka, Necessary conditions of topological conjugacy for threedimensional diffeomorphisms with heteroclinic tangencies, Dynamical Systems 3 (2013), no. 31, issue 3-4, 185-200.

[10] E. A. Leontovich and A. G. Măer, On a scheme defining the topological structure of a partition into trajectories, Dokl. Akad. Nauk SSSR 103 (1955), no. 4, 557-560. (Russian) MR0072305

[11] W. Melo Moduli of stability of two-dimensional diffeomorphisms, Topology 19 (1980), no. 1, 9-21. MR:559473

[12] W. Melo and J. Palis, Moduli of stability for diffeomorphisms, in: Global theory of dynamical systems, Proc. Internat. Conf., Northwestern Univ. Evanston, 1979 (Lecture Notes in Math. vol. 819), Springer, Berlin, 1980, 318-339. MR591192

[13] W. Melo amd S. J. Strien, Diffeomorphisms on surfaces with a finite number of moduli, Ergod. Theory Dynam. Sys. 7 (1987), no. 3, 415-462. MR.912376

[14] T. M. Mitryakova and O. V. Pochinka, Classification of the simplest diffeomorphisms of the sphere $\mathbf{S}^{2}$ with one modulus of stability, Sovrem. Mat. Prilozh. Inst. Kibern. Akad Nauk Gruzii, 54(2008), 99-112; English transl., J. Math. Sci. (N.Y.) 158 (2009), no. 2, 261-275. MR2677139

[15] T. M. Mitryakova and O. V. Pochinka, On necessary and sufficient conditions for the topological conjugacy of surface diffeomorphisms with a finite number of orbits of heteroclinic tangency, Trudy Mat. Inst. Steklova 270 (2010), 198-219; English transl., Proc. Steklov Inst. Math. 270 (2010), 194-215. MR2768947

[16] T. M. Mitryakova and O. V. Pochinka, Towards the problem of classifying diffeomorphisms of surfaces with finitely many moduli of topological conjugacy, Nonlinear Dynamics 6 (2010), no. 1, 91-105. (Russian)

[17] S. Newhouse, J. Palis, and F. Takens, Bifurcations and stability of families of diffeomorphisms, Publ. Math. IHES 57 (1983), no. 1, 5-71. MR699057

[18] J. Palis, A differentiable invariant of topological conjugacies and moduli of stability, Dynamical systems, vol. III (Astérisque, no. 51), Soc. Math. France, Paris, 1978, 335-346. MR0494283

[19] J. Palis and W. de Melo, Geometric theory of dynamical systems. An introduction, Springer Verlag, New York-Heidelberg-Berlin, 1982. MR669541

[20] M. Peixoto, On the classification of flows on 2-manifolds, in: Dynamical Systems, Proc. Symp. Univ. Bahia, Salvador, Brasil, 1971, Acad. Press, New York-London, 1973, 389-419. MR0334289

[21] S. Smale, On gradient dynamical systems, Ann. Math. 74 (1961), 199-206. MR0133139

$[22]$ L. P. Shil'nikov, A. L. Shil'nikov, D. Turaev, and L. O. Chua, Methods of qualitative theory in nonlinear dynamics, Part I, World Scientific Series on Nonlinear Science. Series A: Monographs 
and Treatises, vol. 4, World Scientific, River Edge, NJ, 1998; Part II, World Scientific Series on Nonlinear Science. Series A: Monographs and Treatises, vol. 5, World Scientific, River Edge, NJ, 2001. MR 1691840; MR1884710

Department of Mechanics and Mathematics, Nizhnit Novgorod State University, Nizhmi Novgorod, 603950 Russian Federation

E-mail address: tatiana.mitryakova@yandex.ru

National Research University Higher School of Economics

E-mail address: olga-pochinka@yandex.ru 